\title{
Higher Local Conservation Laws for Nonlinear Sigma Models on Symmetric Spaces
}

\author{
Harald Eichenherr ${ }^{1}$ and Michael Forger ${ }^{2, \star}$ \\ 1 CERN - Geneva, CH-1211 Geneva 23, Switzerland \\ 2 Fakultät für Physik der Universität Freiburg, D-7800 Freiburg i. Brsg., Federal Republic of \\ Germany
}

\section{Dedicated to the Memory of our Colleague and Friend Jorge André Swieca}

\begin{abstract}
We show that for the classical two-dimensional nonlinear $\sigma$-model on a Riemannian symmetric space of dimension $m$ and rank $p$, there exist $p$ independent series of higher local conservation laws, and we reduce the field equations of the model to a system of nonlinear partial differential equations possessing an associated Lax pair and involving $m+p$ independent variables.
\end{abstract}

\section{Introduction}

This is the third in a series of papers devoted to analyzing the structure of classical two-dimensional nonlinear $\sigma$-models on Riemannian symmetric spaces $M=G / H$. These are field theories of geometric nature which generalize the nonlinear $\sigma$-models on the spheres $S^{N-1}=S O(N) / S O(N-1)$ [1] or the complex projective spaces $\mathbb{C} P^{N-1}=S U(N) / S(U(1) \times U(N-1))$ [2]. Our current interest in them mainly stems from the possibility to study systematically the differential geometric roots of integrability properties.

In our first paper [3, I], we introduced a general formulation of the nonlinear $\sigma$-model on a Riemannian homogeneous space (see also $[4,5]$ ), and we proved it to possess the so-called dual symmetry if (and only if - cf. [6]) the homogeneous space is a symmetric one. This hidden dynamical symmetry yields a linear Lax representation of the nonlinear field equations and leads to an infinite series of nonlocal conservation laws, thus generalizing the corresponding well-known features of the $S^{N-1}$ prototype model [7] or the $\mathbb{C} P^{N-1}$ model [2].

In our second paper [3, II], we used certain structural properties of symmetric spaces to clarify the relation between the principal nonlinear $\sigma$-model on a Lie group $G$ and the nonlinear $\sigma$-models on the symmetric quotient spaces $M=G / H$ for $G$, and to show that instantons are fixed points of the dual symmetry.

Our present work deals with the derivation of higher local conservation laws for the nonlinear $\sigma$-model on an arbitrary Riemannian symmetric space $M=G / H$, generalizing the corresponding well-known higher local conservation laws for the

* Work partially done under DFG contract Schr $4 / 5$ 
$S^{N-1}$ prototype model [1] or the $\mathbb{C} P^{N-1}$ model [8]. As usual, these conservation laws are the coefficients in a power series expansion for a certain one-parameter family of continuity equations, to be extracted from the dual symmetry in a suitable way. For the $S^{N-1}$ prototype model, this was originally done [1] via a one-parameter family of Bäcklund transformations, or equivalently, of matrix Riccati equations, and both methods have been generalized to various other types of nonlinear $\sigma$-models [9-13]. However, the equations used there do not have an intrinsic geometric meaning and hence do not match with the general geometric structure, nor do they, in our opinion, produce all the relevant information.

Fortunately, we can dispense with these additional ingredients and instead, we exhibit directly a one-parameter family of $\mathfrak{a}$-valued fields $a^{(\gamma)}$ which serves as the generating functional of the aforementioned one-parameter family of local continuity equations : this is simply the a-component in a certain decomposition of the one-parameter family of $G$-valued fields $g^{(\gamma)}$ which results from applying the dual symmetry to the original $G$-valued field $g$. (Here and throughout this paper, a denotes a fixed maximal abelian subspace of $m, m$ being the tangent space of $M=G / H$ at the distinguished point $\circ=1 H$; cf. Sect. 2.) The differential equations for the $g^{(\gamma)}$ then lead to differential equations for the $a^{(\gamma)}$ which, when expanded in powers of $\gamma$, can be solved recursively to yield the desired conservation laws. Moreover, it is clear that at each step of the recursion, we obtain $p$ scalar conservation laws rather than just a single one, where by definition, $p=\operatorname{dim} a$ is the rank of $M$.

Although in principle, this program can be carried through in any gauge, we have found it essential in practice to work in the so-called partial reduction gauge (PR gauge) given by ${ }^{1}$

$$
k_{\xi} \equiv g^{-1} D_{\xi} g \text { takes values in } \mathfrak{a} .
$$

This gauge condition on the original $G$-valued field $g$ may always be imposed, as follows from (the infinitesimal version of the polar coordinate decomposition theorem for symmetric spaces (cf. Sect. 2), and it is local. Together with the structure of the recursion relations, this implies the conservation laws to be local. Moreover, the whole construction shows them to be invariant under global symmetry transformations as well as under residual gauge transformations, i.e. gauge transformations preserving the PR gauge condition.

As yet another interesting consequence of the interplay between the structure of symmetric spaces and of nonlinear $\sigma$-models, let us mention the fact that for solutions of the field equations, we can fix the gauge completely and work in the so-called reduction gauge ( $\mathrm{R}$ gauge). More precisely, the corresponding additional gauge condition on the original $G$-valued field $g$ (which, by the way, is nonlocal) removes the gauge freedom completely up to global residual gauge transformations. This reduction procedure leads to a definition of the so-called reduced system as a system of nonlinear partial differential equations in $m+p$ variables, or if one also exploits the conformal invariance of the theory and introduces normalized coordinates to eliminate two more degrees of freedom, in $m+p-2$ variables, where $m=\operatorname{dim} M, p=\operatorname{rank} M$. These reduced systems, at least for rank 1

1 Actually, the PR gauge condition is slightly more restrictive; cf. Sect. 3 
spaces (such as the spheres $S^{N-1}$ and the complex projective spaces $\mathbb{C} P^{N-1}$ ) and in normalized coordinates, can be viewed as generalizations of the sine-Gordon equation. Unfortunately, except in a few very special cases $\left(M=S^{2}=\mathbb{C} P^{1}, M=S^{3}\right.$, $M=\mathbb{C} P^{2}-$ cf. $\left.[1,14]\right)$, it remains unclear whether these differential equations possibly after an appropriate transformation of variables - can be derived from a Lagrangian and hence define a field theory. Still, the existence of a Lax pair, together with the examples and with the fact that they possess higher local conservation laws - namely just the ones for the original $\sigma$-models, leads us to conjecture that they actually define completely integrable systems which can be solved by the inverse scattering method.

The organization of the paper is as follows: In Sect. 2, we give a brief account of the basic concepts and facts from the theory of Riemannian symmetric spaces. Section 3 reviews the formulation of the classical two-dimensional nonlinear $\sigma$-model on such a space, and Sect. 4 is devoted to the reduction procedure. In Sect. 5, we recall the definition of the dual symmetry and describe the technique of extracting local conservation laws, while in the final Sect. 6, we illustrate our results, together with the general mathematical machinery, for the example of the nonlinear $\sigma$-models on the complex Grassmannian $S U(N) / S(U(p) \times U(q))$ and on the real Grassmannian $S O(N) / S O(p) \times S O(q)(N=p+q, p \leqq q)$.

\section{Riemannian Symmetric Spaces}

For our analysis of nonlinear $\sigma$-models on Riemannian symmetric spaces, we shall introduce, first of all, a certain amount of terminology and notation which we have freely assembled from the previous papers [3] and from the books of Helgason [15] and Kobayashi and Nomizu [16]. Despite the risk of boring the reader, we believe this to be necessary because we will use considerably more detailed information than is contained in [3] and because we have found it convenient to modify some of the notation; also, it may be difficult for the reader to extract the relevant facts from the enormous amount of information contained in the mathematical literature $[15,16]$.

Throughout this paper, $M=G / H$ will denote a Riemannian globally symmetric space, and without much loss of generality [3, II], we shall assume it to be either of the compact type or of the noncompact type. This means that we may take $G$ to be a connected semisimple Lie group with Lie algebra $g$ and $H \subset G$ to be a closed subgroup with Lie algebra $\mathfrak{h} C \mathfrak{g}$ such that the group of linear transformations on $\mathfrak{g}$ of the form $\operatorname{Ad}(h), h \in H$, is compact. For simplicity, we also assume $G$ to be simply connected if $M$ is of the compact type and to have finite center if $M$ is of the noncompact type; then in both cases, $H$ itself will be compact and connected [15, pp. 320/321 and 252/253]. We write $\sigma$ for the involutive automorphism of $G$ which defines the symmetry around the distinguished point $\circ \in M$ ( $=$ the left coset $H=1 H$ of $1 \in G$ ) and $\dot{\sigma}$ for the involutive automorphism of $g$ which is the derivative of $\sigma$ at $1 \in G$, so that

$$
\sigma(\exp X)=\exp \dot{\sigma}(X) \text { for } X \in \mathfrak{g} ;
$$

recall that the term "involutive automorphism" simply means that

$$
\sigma^{2}=\mathrm{id}_{G}, \quad \sigma\left(g_{1} g_{2}\right)=\sigma\left(g_{1}\right) \sigma\left(g_{2}\right) \text { for } g_{1}, g_{2} \in G
$$


for $\sigma$ and

$$
\dot{\sigma}^{2}=\mathrm{id}_{\mathfrak{g}}, \quad \dot{\sigma}\left(\left[X_{1}, X_{2}\right]\right)=\left[\dot{\sigma} X_{1}, \dot{\sigma} X_{2}\right] \text { for } X_{1}, X_{2} \in \mathfrak{g}
$$

for $\dot{\sigma}$. Then $H$ is precisely the fixed point set of $\sigma, \mathfrak{h}$ is precisely the fixed point set of $\dot{\sigma}$, and $m$, the eigenspace of $\dot{\sigma}$ belonging to the eigenvalue -1 , can (and will) be identified with the tangent space $T_{\circ} M$ of $M$ at the distinguished point $\circ \in M$; moreover, the first formula in (2.3) yields the direct decomposition

$$
\mathfrak{g}=\mathfrak{h} \oplus \mathfrak{m} \quad \text { with } \quad \dot{\sigma}=+1 \text { on } \mathfrak{h}, \quad \dot{\sigma}=-1 \text { on } \mathfrak{m},
$$

and the second formula in (2.3) is equivalent to the by now familiar commutation relations

$$
[\mathfrak{h}, \mathfrak{h}] \subset \mathfrak{h}, \quad[\mathfrak{h}, \mathfrak{m}] \subset \mathfrak{m}, \quad[\mathfrak{m}, \mathfrak{m}] \subset \mathfrak{h} .
$$

Furthermore, there exists an $\operatorname{Ad}(G)$-invariant nondegenerate symmetric bilinear form $(\cdot, \cdot)$ on $\mathfrak{g}$ (satisfying $\mathfrak{h} \perp \mathfrak{m}$ ) which restricts to an $\mathrm{Ad}(H)$-invariant positive definite symmetric bilinear form $(\cdot, \cdot)$ on $m$; in fact, we may just choose $(\cdot, \cdot)$ on $\mathfrak{g}$ to be some negative (positive) multiple of the Killing form of $\mathfrak{g}$ if $M$ is of the compact (noncompact) type [15, pp. 231 ff.]. (For an analysis of the situation on a more general class of homogeneous spaces, see [6].) Of course, using the respective actions of $G$ on (the tangent bundles of) $G$ and $M$ by left translations, the forms $(\cdot, \cdot)$ on $g$ and on $m$ can be uniquely extended to a biinvariant pseudo-Riemannian metric on $G$ and to a left invariant Riemannian metric on $M$, respectively; both of these will also be simply denoted by $(\cdot, \cdot)$. Thus $M$ becomes a Riemannian manifold whose geodesics turn out to be given by the projection of certain oneparameter subgroups of $G$ to $M$; more precisely, for $g \in G, g H=m \in M$, and $X \in \mathrm{m}$, the geodesic $\gamma=\gamma(t)$ on $M$ starting at $\gamma(0)=m \in M$ with initial velocity $\dot{\gamma}(0)$ $=g X \in T_{m} M$ is given by $\gamma(t)=g \exp (t X) H$ for $t \in \mathbb{R}$. Thus the exponential mapping

$$
\operatorname{Exp}: \mathfrak{m} \rightarrow M
$$

for $M$ at $\circ$ in the sense of Riemannian geometry [15, pp. 32/33 and 55-58] and the exponential mapping

$$
\exp : \mathfrak{g} \rightarrow G
$$

for $G$ in the sense of Lie group theory are simply related by

$$
\operatorname{Exp}(X)=\exp (X) H \quad \text { for } X \in \mathfrak{m} \text {; }
$$

in particular, $M$ is complete, and Exp maps $m$ onto $M$. If $M$ is of the noncompact type, Exp is even a global diffeomorphism of $m$ onto $M$ [15, pp. 252/253].

Now let us fix once and for all a maximal abelian subspace $\mathfrak{a}$ in $\mathrm{m}$ ( $p=\operatorname{dim} \mathfrak{a}$ being the rank of $M)$ together with a maximal abelian subalgebra $\mathfrak{f}$ in $\mathfrak{g}(r=\operatorname{dim} \mathfrak{f}$ being the rank of $G$ ), and write

$$
\begin{gathered}
L^{\prime}=\text { normalizer of } \mathfrak{a} \text { in } H=\{h \in H / \operatorname{Ad}(h) \mathfrak{a}=\mathfrak{a}\} \\
L=\text { centralizer of } \mathfrak{a} \text { in } H=\{h \in H / \operatorname{Ad}(h)=1 \text { on } \mathfrak{a}\} \\
\mathfrak{l}=\text { centralizer of } \mathfrak{a} \text { in } \mathfrak{h}=\{X \in \mathfrak{h} / \operatorname{ad}(X)=0 \text { on } \mathfrak{a}\} .
\end{gathered}
$$


The geometric interpretation of the subspace $a$ comes from the fact that $\operatorname{Exp}(\mathfrak{a})$ is a maximal flat totally geodesic submanifold of $M$ - the direct analogue of a maximal torus [15, pp. 224/225 and 245-249]. Both $L^{\prime}$ and $L$ are closed subgroups of $H$ which have the same Lie algebra $\mathbb{I}$, and the discrete factor group $L^{\prime} / L-$ conveniently viewed as a finite group of linear transformations on $\mathfrak{a}$ - is the Weyl group $W(M)$ of $M[15$, pp. 284 and 401/402]. Also, we define the inhomogeneous or affine Weyl group $I W(M)$ of $M$ to be the semidirect product of $W(M)$ with the discrete group of translations in $\mathfrak{a}$ by vectors on the lattice

$$
\{X \in \mathfrak{a} / \operatorname{Exp}(X)=\circ\}=\{X \in \mathfrak{a} / \exp (X) \in H\}=\{X \in \mathfrak{a} / \exp (2 X)=1\}
$$

in $\mathfrak{a}[15, \mathrm{pp} .321 / 322]$. [Observe that this lattice is trivial, and $I W(M)=W(M)$, if $M$ is of the noncompact type.] Now letting $\mathfrak{p}$ denote the orthogonal complement of $\mathfrak{I}$ in $\mathfrak{h}$ and $\mathfrak{n}$ denote the orthogonal complement of $\mathfrak{a}$ in $\mathfrak{m}$ with respect to the form $(\cdot, \cdot)$, we arrive at the orthogonal direct decomposition

$$
\mathfrak{g}=\mathfrak{h} \oplus \mathfrak{m}=\mathfrak{l} \oplus \mathfrak{p} \oplus \mathfrak{a} \oplus \mathfrak{n},
$$

and the corresponding decomposition of elements $X \in \mathfrak{g}$ will be written in the form

$$
X=X_{\mathfrak{h}}+X_{\mathfrak{m}}=X_{\mathrm{I}}+X_{\mathfrak{p}}+X_{\mathfrak{a}}+X_{\mathfrak{n}} .
$$

The commutation relations (2.5) can then be further specified as follows:

$$
\begin{gathered}
{[\mathfrak{l}, \mathfrak{l}] \subset \mathfrak{l}, \quad[\mathfrak{l}, \mathfrak{p}] \subset \mathfrak{p}, \quad[\mathfrak{p}, \mathfrak{p}] \subset \mathfrak{l} \oplus \mathfrak{p}} \\
{[\mathfrak{l}, \mathfrak{a}]=\{0\}, \quad[\mathfrak{l}, \mathfrak{n}] \subset \mathfrak{n}, \quad[\mathfrak{p}, \mathfrak{a}] \subset \mathfrak{n}, \quad[\mathfrak{p}, \mathfrak{n}] \subset \mathfrak{a} \oplus \mathfrak{n}} \\
{[\mathfrak{a}, \mathfrak{a}]=\{0\}, \quad[\mathfrak{a}, \mathfrak{n}] \subset \mathfrak{p}, \quad[\mathfrak{n}, \mathfrak{n}] \subset \mathfrak{l} \oplus \mathfrak{p} .}
\end{gathered}
$$

Using a superscript ${ }^{c}$ to denote complexifications and extending the form $(\cdot, \cdot)$ in a complex bilinear way, so that (2.5) and (2.11)-(2.13) continue to hold (with the obvious notational modifications), we see that $\mathfrak{g}^{c}$ is semisimple, and $\mathfrak{f}^{c}$ is a Cartan subalgebra of $\mathfrak{g}^{c}[15$, p. 259], so that we can define the corresponding system $\Delta$ of (nonzero) roots $\alpha$ on $\mathfrak{f}^{c}[15, \mathrm{pp} .165 \mathrm{ff}]$ and the corresponding system $\Sigma$ of (nonzero) restricted roots $\lambda=\bar{\alpha}$ on $\mathfrak{a}^{c}[15, \mathrm{pp} .263 / 264]$ : We view roots $\alpha$ as linear forms on $\mathfrak{f}^{c}$ and restricted roots $\lambda=\bar{\alpha}$ as linear forms on $\mathfrak{a}^{c}$, where the bar denotes restriction of linear forms from $\mathfrak{f}^{c}$ to $\mathfrak{a}^{c}$; then $\alpha$ will be imaginary (real) on $\mathfrak{f}$ and $\lambda=\bar{\alpha}$ will be imaginary (real) on $\mathfrak{a}$ if $M$ is of the compact (noncompact) type. The multiplicity $m(\lambda)$ of a restricted root $\lambda \in \Sigma$ is the number of roots $\alpha \in \Delta$ whose restriction $\bar{\alpha}$ is just $\lambda$. The set $\Sigma$ of restricted roots gives rise to two important subsets of $\mathfrak{a}$ of measure zero, namely

$$
D^{\circ}(M)=\{X \in \mathfrak{a} / \lambda(X)=0 \text { for some } \lambda \in \Sigma\},
$$

which is the union of finitely many hyperplanes in $\mathfrak{a}$, and

$$
D(M)=\{X \in \mathfrak{a} / \lambda(X) \in \pi i \mathbb{Z} \text { for some } \lambda \in \Sigma\},
$$

which is the union of finitely many families of evenly spaced affine hyperplanes in $\mathfrak{a}$; the latter is called the diagram of $M\left[15\right.$, p. 295]. The complement $\mathfrak{a} \backslash D^{\circ}(M)$ of $D^{\circ}(M)$ in a decomposes into a finite number of connected components called Weyl chambers, while the complement $\mathfrak{a} \backslash D(M)$ of $D(M)$ in $\mathfrak{a}$ decomposes into a countable 
number of connected components called Weyl cells. [Observe that $D(M)=D^{\circ}(M)$, and the cells are identical with the chambers, if $M$ is of the noncompact type.] As it turns out, the Weyl group of $M$ is generated by the reflections in the walls of the Weyl chambers and just permutes these, while the affine Weyl group of $M$ is generated by the reflections in the walls of the Weyl cells and just permutes these [15, pp. 288/289 and 321/322]. We also write $\mathfrak{a}_{s}^{\circ}\left(\mathfrak{a}_{s}\right)$ for $D^{\circ}(M)[D(M)]$ and $\mathfrak{a}_{r}^{\circ}\left(\mathfrak{a}_{r}\right)$ for its complement. Then under the smooth map

$$
\begin{gathered}
H \times \mathfrak{a} \rightarrow \mathfrak{m} \\
(h, X) \mapsto \operatorname{Ad}(h) X
\end{gathered}
$$

of $H \times \mathfrak{a}$ onto $\mathfrak{m}$ [15, pp. 246-248 and 294/295], $H \times \mathfrak{a}_{s}^{\circ}$ respectively $H \times \mathfrak{a}_{r}^{\circ}$ is mapped to what is called the singular set $\mathrm{m}_{s}^{\circ}$ respectively the regular set $\mathrm{m}_{r}^{\circ}$ in $\mathrm{m}$, and similarly, under the smooth map

$$
\begin{gathered}
H \times \mathfrak{a} \rightarrow M \\
(h, X) \mapsto \operatorname{Exp}(\operatorname{Ad}(h) X)=h \operatorname{Exp}(X)
\end{gathered}
$$

of $H \times \mathfrak{a}$ onto $M$ [15, pp. 246-248 and 294/295], $H \times \mathfrak{a}_{s}$ respectively $H \times \mathfrak{a}_{r}$ is mapped to what is called the singular set $M_{s}$ respectively the regular set $M_{r}$ in $M$.

With all this notation, we can state the

\section{Polar Coordinate Decomposition Theorem for Symmetric Spaces}

a) Fix a Weyl chamber $\mathfrak{a}^{+}$in $\mathfrak{a}$. Then every point $Z \in \mathfrak{m}$ can be written in the form

$$
Z=\operatorname{Ad}(h) X
$$

with $h \in H$ and an element $X \in \mathfrak{a}$ which is uniquely determined up to the action of an arbitrary element of the Weyl group $W(M)$, and is uniquely determined if in addition, $X \in \overline{\mathfrak{a}^{+}}$. Moreover, if $Z$ is regular, i.e. $Z \in \mathfrak{m}_{r}^{\circ}$, then $h$ is uniquely determined up to multiplication from the right by an arbitrary element of $L^{\prime}$, and of $L$ if in addition, $X \in \mathfrak{a}^{+}$. Thus the smooth map

$$
\begin{gathered}
H / L \times \mathfrak{a} \rightarrow \mathfrak{m} \\
(h L, X) \mapsto \operatorname{Ad}(h) X
\end{gathered}
$$

of $H / L \times \mathfrak{a}$ onto $\mathfrak{m}$ induced by (2.16) restricts to a local diffeomorphism

$$
H / L \times \mathfrak{a}_{r}^{\circ} \rightarrow \mathrm{m}_{r}^{\circ}
$$

and even to a global diffeomorphism

$$
H / L \times \mathfrak{a}^{+} \stackrel{\cong}{\longrightarrow} \mathrm{m}_{r}^{\circ} .
$$

b) Fix a Weyl cell $\mathfrak{c}^{+}$in $\mathfrak{a}$, contained in some Weyl chamber $\mathfrak{a}^{+}$in $\mathfrak{a}$, whose closure $\bar{c}^{+}$contains the origin. Then every point $m \in M$ can be written in the form

$$
m=\operatorname{Exp}(\operatorname{Ad}(h) X)=h \operatorname{Exp}(X)
$$

with $h \in H$ and an element $X \in \mathfrak{a}$ which is uniquely determined up to the action of an arbitrary element of the affine Weyl group $I W(M)$ of $M$, and is uniquely determined 
if in addition, $X \in \overline{\mathfrak{c}^{+}}$. Moreover, if $m$ is regular, i.e. $m \in M_{r}$, then $h$ is uniquely determined up to multiplication from the right by an arbitrary element of $L^{\prime}$, and of $L$ if in addition, $X \in \mathrm{c}^{+}$. Thus the smooth map

$$
\begin{gathered}
H / L \times \mathfrak{a} \rightarrow M \\
(h L, X) \mapsto \operatorname{Exp}(\operatorname{Ad}(h) X)=h \operatorname{Exp}(X)
\end{gathered}
$$

of $H / L \times \mathfrak{a}$ onto $M$ induced by (2.17) restricts to a local diffeomorphism

$$
H / L \times \mathfrak{a}_{r} \rightarrow M_{r}
$$

and even to a global diffeomorphism

$$
H / L \times \mathfrak{c}^{+} \stackrel{\cong}{\longrightarrow} M_{r} .
$$

For proofs, see [15, pp. 246-248, 285, 293-295, 323/324, and 401-403].

For the sake of definiteness, let us also introduce a basis $K_{1}, \ldots, K_{p}$ of $\mathfrak{a}$ and extend it to a basis $K_{1}, \ldots, K_{p}, K_{p+1}, \ldots, K_{r}$ of $\mathfrak{f}$; of course, these basis vectors are assumed to be orthonormal with respect to the form $(\cdot, \cdot)$ on $\mathfrak{g}$, i.e. we have

$$
\begin{gathered}
\left(K_{i}, K_{i}\right)=+1 \text { for } 1 \leqq i \leqq p \\
\left(K_{i}, K_{i}\right)=+1 \text { for } p+1 \leqq i \leqq r \quad \text { (compact type) } \\
\left(K_{i}, K_{i}\right)=-1 \text { for } p+1 \leqq i \leqq r \quad \text { (noncompact type) }
\end{gathered}
$$

In other words, with respect to the Killing form Kill of $\mathfrak{g}^{c}$, we have

$$
\begin{gathered}
\operatorname{Kill}\left(K_{i}, K_{i}\right)=-c^{2} \text { for } 1 \leqq i \leqq p \quad \text { (compact type), } \\
\operatorname{Kill}\left(K_{i}, K_{i}\right)=+c^{2} \text { for } 1 \leqq i \leqq p \quad \text { (noncompact type), } \\
\operatorname{Kill}\left(K_{i}, K_{i}\right)=-c^{2} \text { for } p+1 \leqq i \leqq r
\end{gathered}
$$

where $c>0$ is some suitable constant. This basis of $\mathfrak{f}^{c}$ is further extended to a Cartan basis of $\mathfrak{g}^{c}$ by introducing root vectors $E_{\alpha} \in \mathfrak{g}^{c}, \alpha \in \Delta$, such that defining $K_{\alpha} \in \mathfrak{H}^{c}, \alpha \in \Delta$, by

$$
\operatorname{Knll}\left(K_{\alpha}, K\right)=\alpha(K) \text { for all } K \in \mathfrak{l}^{c},
$$

the commutation relations for $\mathfrak{g}^{c}$ read

$$
\begin{aligned}
& {\left[K, E_{\alpha}\right]=} \alpha(K) E_{\alpha} \quad \text { for all } K \in \mathfrak{f}^{c}, \\
& {\left[E_{\alpha}, E_{-\alpha}\right]=K_{\alpha}, } \\
& {\left[E_{\alpha}, E_{\beta}\right]=N_{\alpha, \beta} E_{\alpha+\beta} \text { if } \alpha+\beta \neq 0, }
\end{aligned}
$$

(with $N_{\alpha, \beta}=0$ if $\alpha+\beta \notin \Delta$ ), while the orthogonality relations with respect to the Killing form are

$$
\begin{aligned}
& \operatorname{Kill}\left(K, E_{\alpha}\right)=0 \text { for all } K \in \mathfrak{F}^{c}, \\
& \operatorname{Kill}\left(E_{\alpha}, E_{-\alpha}\right)=1, \\
& \operatorname{Kill}\left(E_{\alpha}, E_{\beta}\right)=0 \text { if } \alpha+\beta \neq 0
\end{aligned}
$$


[15, pp. 166 and 176/177]. Moreover, positivity of roots and of restricted roots and hence the system $\Delta^{+} C \Delta$ and the system $\Sigma^{+} C \Sigma-$ is defined in terms of the given basis of $\mathfrak{f}^{c}$ by the usual "lexicographic ordering" [15, pp. 172/173 and $259 / 260]$, and the Weyl chamber $\mathfrak{a}^{+}$appearing in the theorem above will then always be assumed to be that subset of $a$ on which all the positive (restricted) roots take positive values $[15$, p. 292]. In these terms, the orthogonal direct decomposition (2.11) is given by

$$
\begin{gathered}
\mathfrak{I}^{c}=(\mathfrak{f} \cap \mathfrak{h})^{c} \oplus \sum_{\substack{\alpha \in \Delta^{+} \\
\bar{\alpha}=0}} \mathbb{C} E_{\alpha} \oplus \sum_{\substack{\alpha \in \Delta^{+} \\
\bar{\alpha}=0}} \mathbb{C} E_{-\alpha} \\
\mathfrak{p}^{c}=\sum_{\substack{\alpha \in \Delta^{+} \\
\bar{\alpha} \neq 0}} \mathbb{C}\left(E_{\alpha}+\dot{\sigma} E_{\alpha}\right) \\
\mathfrak{n}^{c}=\sum_{\substack{\alpha \in \Delta^{+} \\
\bar{\alpha} \neq 0}} \mathbb{C}\left(E_{\alpha}-\dot{\sigma} E_{\alpha}\right) .
\end{gathered}
$$

Moreover, for positive integers $n$ and all $K \in \mathfrak{a}$, the linear transformation $\operatorname{ad}(K)^{n}$ takes $\mathfrak{p}^{c}$ to $\mathfrak{p}^{c}$ respectively to $\mathfrak{n}^{c}$ and $\mathfrak{n}^{c}$ to $\mathfrak{n}^{c}$ respectively to $\mathfrak{p}^{c}$ if $n$ is even respectively odd; explicitly, it is given by

$$
\operatorname{ad}(K)^{n}\left(\sum_{\substack{\alpha \in \Delta^{+} \\ \bar{\alpha} \neq 0}} X^{\alpha}\left(E_{\alpha} \pm \dot{\sigma} E_{\alpha}\right)\right)=\sum_{\substack{\alpha \in \Delta^{+} \\ \bar{\alpha} \neq 0}} X^{\alpha} \alpha(K)^{n}\left(E_{\alpha} \pm(-1)^{n} \dot{\sigma} E_{\alpha}\right) .
$$

This shows that if $K$ is regular, i.e. if $K \in \mathfrak{a}_{r}^{\circ}$, then for any integer $n$ (positive or negative), $\operatorname{ad}(K)^{n}$ is an invertible linear transformation on the space $\mathfrak{p}^{c} \oplus \mathfrak{n}^{c}$ which acts according to (2.32) and commutes with the action of $\operatorname{Ad}(L)$ and $\operatorname{ad}(l)$, i.e.

$$
\begin{array}{clll}
\operatorname{ad}(K)^{n} \operatorname{Ad}(h) X=\operatorname{Ad}(h) \operatorname{ad}(K)^{n} X & \text { for } & h \in L, & X \in \mathfrak{p}^{c} \oplus \mathfrak{n}^{c}, \\
\operatorname{ad}(K)^{n}[Z, X]=\left[Z, \operatorname{ad}(K)^{n} X\right] & \text { for } & Z \in \mathfrak{l}, & X \in \mathfrak{p}^{c} \oplus \mathfrak{n}^{c} .
\end{array}
$$

\section{Formulation of Nonlinear $\sigma$-Models}

With the notations and conventions of Sect. 2, we shall now review briefly the formulation of the classical two-dimensional nonlinear $\sigma$-model on $M$, where as usual $[3, I]$, the field $q=q(x)$ taking values in $M=G / H$ is (locally) lifted to a field $g=g(x)$ taking values in $G$, subject to the natural gauge equivalence

$$
g_{2}(x) \sim g_{1}(x) \Leftrightarrow q_{2}(x)=q_{1}(x) \Leftrightarrow \begin{aligned}
& \text { There exists a field } \\
& h=h(x) \text { taking values } \\
& \text { in } H \text { such that } \\
& g_{2}(x)=g_{1}(x) h(x)
\end{aligned}
$$

under H. Still following [3, I], we consider the (left translated) derivative field $g^{-1} \partial_{\mu} g$ (taking values in $\mathrm{g}$ ) and split it into its vertical part, which is the gauge field $A_{\mu}$ (taking values in $\mathfrak{h}$ ), and its horizontal part, which is the (left translated) covariant derivative field $k_{\mu} \equiv g^{-1} D_{\mu} g$ (taking values in $\mathrm{m}$ ):

$$
A_{\mu}=\left(g^{-1} \partial_{\mu} g\right)_{\mathfrak{h}}, \quad k_{\mu} \equiv g^{-1} D_{\mu} g=\left(g^{-1} \partial_{\mu} g\right)_{\mathfrak{m}} .
$$


We also introduce the Noether current ${ }^{2} j_{\mu}$ (taking values in $\mathrm{g}$ ):

$$
j_{\mu}=-D_{\mu} g g^{-1}
$$

[cf. (2.12) for the notation]. Indeed, it follows from the $\operatorname{Ad}(H)$-invariance of the direct decomposition $\mathfrak{g}=\mathfrak{h} \oplus \mathfrak{m}$ [cf. (2.11)] that under gauge transformations $g \rightarrow g h, j_{\mu}$ is invariant, $A_{\mu}$ transforms as a gauge field, and $k_{\mu}$ is covariant (i.e. $j_{\mu} \rightarrow j_{\mu}$, $A_{\mu} \rightarrow h^{-1} A_{\mu} h+h^{-1} \partial_{\mu} h$, and $\left.k_{\mu} \rightarrow h^{-1} k_{\mu} h\right)$, which motivates the introduction of an $H$-covariant derivative for the latter:

$$
D_{\mu} k_{v}=\partial_{\mu} k_{v}+\left[A_{\mu}, k_{v}\right] \text {. }
$$

Then as a consequence of the symmetric space structure of $M$, the identities

$$
\begin{gathered}
F_{\mu \nu} \equiv \partial_{\mu} A_{\nu}-\partial_{\nu} A_{\mu}+\left[A_{\mu}, A_{v}\right]=-\left[k_{\mu}, k_{v}\right], \\
\partial_{\mu} j_{\nu}-\partial_{v} j_{\mu}+\left[j_{\mu}, j_{v}\right]=0, \\
D_{\mu} k_{\nu}-D_{\nu} k_{\mu}=0
\end{gathered}
$$

hold for any field configuration; in fact, according to (2.5), the first respectively third equation is just the vertical part (h-component) respectively horizontal part (m-component) of the identity

$$
\partial_{\mu}\left(g^{-1} \partial_{\nu} g\right)+g^{-1} \partial_{\mu} g g^{-1} \partial_{\nu} g-\partial_{\nu}\left(g^{-1} \partial_{\mu} g\right)-g^{-1} \partial_{\nu} g g^{-1} \partial_{\mu} g=0
$$

and the proof of the second equation is similar. Moreover, following $[3, \mathrm{II}]$, we introduce a field $Q=Q(x)$ taking values in $G$, as follows:

$$
Q=\sigma(g) g^{-1}
$$

Obviously, $Q$ is gauge invariant, and we obtain

$$
j_{\mu}=\frac{1}{2} Q^{-1} \partial_{\mu} Q \text {. }
$$

In the following, we shall often subject the field $g=g(x)$ to gauge transformations $g \rightarrow g h$ with fields $h=h(x)$ taking values in $L C H$ rather than in all of $H$, and we shall refer to these as residual gauge transformations (because they preserve a certain gauge condition to be specified in the next section). Under these circumstances, it is convenient to further decompose the fields $A_{\mu}$ and $k_{\mu}$ as follows :

$$
A_{\mu}=\left(A_{\mu}\right)_{\mathrm{I}}+\left(A_{\mu}\right)_{\mathfrak{p}}, \quad k_{\mu}=\left(k_{\mu}\right)_{a}+\left(k_{\mu}\right)_{\mathfrak{r}}
$$

[cf. (2.12) for the notation]. Indeed, it follows from the $\operatorname{Ad}(L)$-invariance of the direct decomposition $\mathfrak{g}=\mathfrak{l} \oplus \mathfrak{p} \oplus \mathfrak{a} \oplus \mathfrak{n}[\mathrm{cf}$. (2.11)] that under residual gauge transformations $g \rightarrow g h,\left(k_{\mu}\right)_{\mathbf{a}}$ is invariant, $\left(A_{\mu}\right)_{I}$ transforms as a gauge field, and $\left(A_{\mu}\right)_{\mathfrak{p}}, \quad\left(k_{\mu}\right)_{\mathfrak{n}}$ are covariant $\quad\left[\right.$ i.e. $\quad\left(k_{\mu}\right)_{\mathfrak{a}} \rightarrow\left(k_{\mu}\right)_{\mathfrak{a}}, \quad\left(A_{\mu}\right)_{\mathfrak{I}} \rightarrow h^{-1}\left(A_{\mu}\right)_{\mathfrak{I}} h+h^{-1} \partial_{\mu} h$, and $\left.\left(A_{\mu}\right)_{\mathfrak{p}} \rightarrow h^{-1}\left(A_{\mu}\right)_{\mathfrak{p}} h,\left(k_{\mu}\right)_{\mathfrak{n}} \rightarrow h^{-1}\left(k_{\mu}\right)_{\mathfrak{n}} h\right]$, which motivates the introduction of an

2 For the proof of the fact that (3.3) does indeed define the Noether current of the nonlinear $\sigma$-model on $M$, as defined by the action functional (3.10), with respect to the global symmetry transformation $q \rightarrow g_{\circ} q, g \rightarrow g_{\circ} g$ with space-time-independent group elements $g_{\circ} \in G$, see [6] 
$L$-covariant derivative for the latter:

$$
\begin{gathered}
D_{\mu}\left(A_{v}\right)_{\mathfrak{p}}=\partial_{\mu}\left(A_{v}\right)_{\mathfrak{p}}+\left[\left(A_{\mu}\right)_{\mathfrak{l}},\left(A_{\nu}\right)_{\mathfrak{p}}\right], \\
D_{\mu}\left(k_{v}\right)_{\mathfrak{n}}=\partial_{\mu}\left(k_{v}\right)_{\mathfrak{n}}+\left[\left(A_{\mu}\right)_{\mathfrak{Y}}\left(k_{v}\right)_{\mathfrak{n}}\right] .
\end{gathered}
$$

The classical two-dimensional nonlinear $\sigma$-model on $M$ is defined in terms of its action functional

$$
S=\frac{1}{2} \int d^{2} x\left(\partial_{\mu} q, \partial^{\mu} q\right)=\frac{1}{2} \int d^{2} x\left(D_{\mu} g, D^{\mu} g\right),
$$

which by the usual variational principle leads to the field equations

$$
D_{\mu} D^{\mu} g-D_{\mu} g g^{-1} D^{\mu} g=0 \text {. }
$$

These imply that $j_{\mu}$ is conserved and $k_{\mu}$ is covariantly conserved, i.e.

$$
\begin{gathered}
\partial_{\mu} j^{\mu}=0, \\
D_{\mu} k^{\mu}=0,
\end{gathered}
$$

and conversely, both (3.12) and (3.13) imply (3.11) [6].

In terms of (local) coordinates $\xi, \eta$ which are complex coordinates in the Euclidean case and light-cone coordinates in the Minkowski case $[3, I]$, the definition (3.2) implies

$$
\begin{aligned}
& \partial_{\xi} g=g\left(A_{\xi}+k_{\xi}\right), \\
& \partial_{\eta} g=g\left(A_{\eta}+k_{\eta}\right),
\end{aligned}
$$

the identities (3.5) take the form

$$
\begin{gathered}
F_{\xi \eta} \equiv \partial_{\xi} A_{\eta}-\partial_{\eta} A_{\xi}+\left[A_{\xi}, A_{\eta}\right]=-\left[k_{\xi}, k_{\eta}\right], \\
\partial_{\xi} j_{\eta}-\partial_{\eta} j_{\xi}+2\left[j_{\xi}, j_{\eta}\right]=0 \\
D_{\xi} k_{\eta}-D_{\eta} k_{\xi}=0
\end{gathered}
$$

the action functional (3.10) can be rewritten as

$$
S=\frac{1}{2} \int d \xi d \eta\left(\partial_{\xi} q, \partial_{\eta} q\right)=\frac{1}{2} \int d \xi d \eta\left(D_{\xi} g, D_{\eta} g\right),
$$

the field equations (3.11) read

$$
D_{\xi} D_{\eta} g-D_{\xi} g g^{-1} D_{\eta} g=0,
$$

or equivalently,

$$
D_{\eta} D_{\xi} g-D_{\eta} g g^{-1} D_{\xi} g=0,
$$

the conservation law (3.12) is

$$
\partial_{\xi} j_{\eta}+\partial_{\eta} j_{\xi}=0
$$

and the covariant conservation law (3.13) becomes

$$
D_{\xi} k_{\eta}=0 \text {, }
$$


or equivalently,

$$
D_{\eta} k_{\xi}=0 \text {. }
$$

On various occasions, we shall find it convenient to decompose the first identity in (3.15) into its I-part and its p-part [cf. (2.13) and (3.9)]:

$$
\begin{gathered}
\partial_{\xi}\left(A_{\eta}\right)_{\mathrm{I}}-\partial_{\eta}\left(A_{\xi}\right)_{\mathrm{I}}+\left[\left(A_{\xi}\right)_{\mathfrak{I}},\left(A_{\eta}\right)_{\mathrm{I}}\right]+\left[\left(A_{\xi}\right)_{\mathfrak{p}},\left(A_{\eta}\right)_{\mathfrak{p}}\right]_{\mathrm{I}}=-\left[k_{\xi}, k_{\eta}\right]_{\mathrm{I}}, \\
D_{\xi}\left(A_{\eta}\right)_{\mathfrak{p}}-D_{\eta}\left(A_{\xi}\right)_{\mathfrak{p}}=-\left[k_{\xi}, k_{\eta}\right]_{\mathfrak{p}} .
\end{gathered}
$$

Similarly, we can decompose the covariant conservation laws (3.20) and (3.21) into their a-part and their n-part [cf. (2.13) and (3.9)]:

$$
\begin{gathered}
\partial_{\xi}\left(k_{\eta}\right)_{\mathfrak{a}}+\left[\left(A_{\xi}\right)_{\mathfrak{p}},\left(k_{\eta}\right)_{\mathfrak{n}}\right]_{\mathfrak{a}}=0, \\
D_{\xi}\left(k_{\eta}\right)_{\mathfrak{n}}+\left[\left(A_{\xi}\right)_{\mathfrak{p}},\left(k_{\eta}\right)_{\mathfrak{a}}\right]+\left[\left(A_{\xi}\right)_{\mathfrak{p}},\left(k_{\eta}\right)_{\mathfrak{n}}\right]_{\mathfrak{n}}=0, \\
\partial_{\eta}\left(k_{\xi}\right)_{\mathfrak{a}}+\left[\left(A_{\eta}\right)_{\mathfrak{p}},\left(k_{\xi}\right)_{\mathfrak{n}}\right]_{\mathfrak{a}}=0, \\
D_{\eta}\left(k_{\xi}\right)_{\mathfrak{n}}+\left[\left(A_{\eta}\right)_{\mathfrak{p}},\left(k_{\xi}\right)_{\mathfrak{a}}\right]+\left[\left(A_{\eta}\right)_{\mathfrak{p}},\left(k_{\xi}\right)_{\mathfrak{n}}\right]_{\mathfrak{n}}=0 .
\end{gathered}
$$

\section{The Reduction Procedure}

The formulation of nonlinear $\sigma$-models given in Sect. 3, where the $M$-valued field $q$ is lifted to a $G$-valued field $g$, obviously has a high amount of redundancy which, however, can be reduced, and eventually even be removed altogether, by imposing suitable gauge conditions. To explain how this is done, we shall restrict our attention to field configurations which are regular in the sense that on the domain of space-time under consideration, the fields $k_{\mu}$ take values in the regular set $m_{r}^{\circ}$ in $m$. [Note that due to the $\operatorname{Ad}(H)$-invariance of the singular set $\mathrm{m}_{s}^{\circ}$ and the regular set $\mathrm{m}_{r}^{\circ}$ in $\mathrm{m}$, this requirement is gauge invariant, i.e. it is really a requirement on the field $q$ rather than just on the field $g$. Note also that $m_{r}^{\circ}$ being open and dense in $m$, the points $x$ satisfying $k_{\mu}(x) \in \mathfrak{m}_{r}^{\circ}$ constitute a domain - i.e. an open subset - in space-time which is also dense if the field $q=g H$ is "sufficiently generic", and our assumption simply means that we focus attention on this domain only.] This is the typical restriction to be expected in a derivation of local conservation laws for nonlinear $\sigma$-models, because the $S^{N-1}$ prototype model [1] already shows that such conservation laws involve rational (rather than polynomial) functions of the fields and their derivatives, so one has to impose some condition which keeps the denominators away from zero.

With these preliminaries out of the way, and fixing a Weyl chamber $\mathfrak{a}^{+}$in $\mathfrak{a}$, we shall now introduce the partial reduction gauge, or briefly $P R$ gauge, and the reduction gauge, or briefly $R$ gauge ${ }^{3}$, as follows:

First, part a) of the polar coordinate decomposition theorem for symmetric spaces implies that for arbitrary (regular) field configurations, we can impose the

3 To be more precise, we should speak of the $\mathrm{PR}_{\xi}$ gauge and the $\mathrm{R}_{\xi}$ gauge because obviously, there is also a $\mathrm{PR}_{\eta}$ gauge and an $\mathrm{R}_{\eta}$ gauge [obtained by replacing $k_{\xi}$ by $k_{\eta}$ in (4.1)] which, however, will not be needed in this paper 
PR gauge condition, which reads

$$
k_{\xi} \text { takes values in } \mathfrak{a}_{r}^{\circ},
$$

or even

$$
k_{\xi} \text { takes values in } \mathfrak{a}^{+} \text {. }
$$

In other words, we claim that the given $M$-valued field $q$ can always (locally) be lifted to a $G$-valued field $g$ satisfying (4.1). Indeed, if to begin with, it has been (locally) lifted to a $G$-valued field $g^{\prime}$ in some arbitrary way, then applying the inverse of the diffeomorphism (2.21), followed by projection onto the first component and then by some (local) section of the bundle $H \rightarrow H / L$, to $k_{\xi}^{\prime}$, we see that (locally) there exists an $H$-valued field $h$ such that under the gauge transformation $g^{\prime} \rightarrow g=g^{\prime} h$, we have $k_{\xi}^{\prime} \rightarrow k_{\xi}=h^{-1} k_{\xi}^{\prime} h$ with $k_{\xi}$ taking values in $\mathfrak{a}_{r}^{\circ}$ or even in $\mathfrak{a}^{+}$. This argument also shows that the PR gauge is a local gauge because obviously, the fields $h$ and $g$ are local functionals of the original field $g^{\prime 4}$. On the other hand, it is only a partial gauge because the condition (4.1a) respectively (4.1b) still leaves us with the freedom to perform gauge transformations $g \rightarrow g h$ with arbitrary $L^{\prime}$-valued respectively $L$-valued fields $h$; this is the motivation for the term "residual' gauge transformations" (for the latter) introduced in Sect. 3.

From our regularity assumptions, we see that in the PR $\operatorname{gauge}, \operatorname{ad}\left(k_{\xi}\right)-$ considered as a field of linear transformations from $n$ to $\mathfrak{p}$ and/or from $\mathfrak{p}$ to $\mathfrak{n}$ takes values in the invertible linear transformations because $\lambda\left(k_{\xi}\right) \neq 0$ for all restricted roots $\lambda \in \Sigma$. Therefore, we can define a field $\phi$ (taking values in $\mathfrak{n}$ ) by

$$
\phi=\operatorname{ad}\left(k_{\xi}\right)^{-1}\left(A_{\xi}\right)_{\mathfrak{p}},
$$

and it follows from (2.33) that under residual gauge transformations $g \rightarrow g h, \phi$ is covariant (i.e. $\phi \rightarrow h^{-1} \phi h$ ), which motivates the introduction of an $L$-covariant derivative for $\phi$ :

$$
D_{\mu} \phi=\partial_{\mu} \phi+\left[\left(A_{\mu}\right)_{\mathrm{I}}, \phi\right]
$$

Then for solutions of the field equations, we have the algebraic equations

$$
\begin{aligned}
\left(A_{\xi}\right)_{\mathfrak{p}} & =\left[k_{\xi}, \phi\right], & \left(A_{\eta}\right)_{\mathfrak{p}} & =0, \\
\left(k_{\xi}\right)_{\mathfrak{n}} & =0, & \left(k_{\eta}\right)_{\mathfrak{n}} & =D_{\eta} \phi,
\end{aligned}
$$

the flatness condition

$$
\partial_{\xi}\left(A_{\eta}\right)_{\mathrm{I}}-\partial_{\eta}\left(A_{\xi}\right)_{\mathrm{I}}+\left[\left(A_{\xi}\right)_{\mathrm{I}},\left(A_{\eta}\right)_{\mathrm{I}}\right]=0,
$$

and the following system of partial differential equations:

$$
\begin{gathered}
\partial_{\eta} k_{\xi}=0, \\
\partial_{\eta}\left(\frac{1}{2}\left[\phi,\left[k_{\xi}, \phi\right]\right]\right)_{a}=\partial_{\xi}\left(k_{\eta}\right)_{a}, \\
D_{\eta} D_{\xi} \phi \underset{(4.5)}{=} D_{\xi} D_{\eta} \phi=\left[\left(k_{\eta}\right)_{\mathfrak{a}},\left[k_{\xi}, \phi\right]\right]+\left[D_{\eta} \phi,\left[k_{\xi}, \phi\right]\right]_{\mathfrak{n}} .
\end{gathered}
$$

4 For an extensive discussion of locality properties of gauges, though in a somewhat different context, see [17] 
For the proof, observe first that in the PR gauge, (3.26) becomes (4.6) and (3.27) [after application of $\operatorname{ad}\left(k_{\xi}\right)^{-1}$ ] becomes the equation $\left(A_{\eta}\right)_{\mathfrak{p}}=0$. From (3.22) respectively (3.23), and using (2.13) respectively (2.13) and (2.33), we then derive (4.5) respectively [after application of $\left.\operatorname{ad}\left(k_{\xi}\right)^{-1}\right]$ the equation $\left(k_{\eta}\right)_{\mathfrak{n}}=D_{\eta} \phi$. Inserting all this into (3.24) respectively (3.25), we finally obtain (4.7) respectively (4.8), where we have used (2.13), (4.6), Jacobi's identity and the product rule to write

$$
\begin{aligned}
{\left[D_{\eta} \phi,\left[k_{\xi}, \phi\right]\right]_{\mathfrak{a}}=} & {\left[\partial_{\eta} \phi,\left[k_{\xi}, \phi\right]\right]_{\mathfrak{a}}+\left[\left(A_{\eta}\right),\left[k_{\xi}, \phi\right]\right]_{\mathfrak{a}} } \\
= & \frac{1}{2}\left[\partial_{\eta} \phi,\left[k_{\xi}, \phi\right]\right]_{\mathfrak{a}}+\frac{1}{2}\left[\phi,\left[k_{\xi}, \partial_{\eta} \phi\right]\right]_{\mathfrak{a}} \\
& -\frac{1}{2}\left[k_{\xi}\left[\phi, \partial_{\eta} \phi\right]\right]_{\mathfrak{a}} \\
= & \frac{1}{2} \partial_{\eta}\left[\phi,\left[k_{\xi}, \phi\right]\right]_{\mathfrak{a}} .
\end{aligned}
$$

As we shall see below (in Sect. 5), the Eqs. (4.6) and (4.7) are precisely the zeroth and the first local conservation law for the nonlinear $\sigma$-model!

Next, the flatness condition (4.4) implies that for (regular) solutions of the field equations, we can go one step further and impose the $\mathrm{R}$ gauge condition, which comprises (4.1) and in addition ${ }^{5}$

$$
\left(A_{\xi}^{R}\right)_{1}=0, \quad\left(A_{\eta}^{R}\right)_{1}=0 .
$$

In other words, we claim that the given $M$-valued field $q$ can always (locally) be lifted to a $G$-valued field $g^{R}$ satisfying (4.1) and (4.9). Indeed, if to begin with, it has been (locally) lifted to a $G$-valued field $g$ satisfying (4.1), then the system

$$
\begin{aligned}
& \partial_{\xi} h^{R}=h^{R}\left(A_{\xi}\right)_{\mathrm{I}} \\
& \partial_{\eta} h^{R}=h^{R}\left(A_{\eta}\right)_{\mathrm{I}}
\end{aligned}
$$

of differential equations, whose compatibility condition is precisely (4.5), defines an $L$-valued field $h^{R}$ such that under the residual gauge transformation $g \rightarrow g^{R}$ $=g\left(h^{R}\right)^{-1}$, we have $k_{\xi} \rightarrow k_{\xi}^{R}=k_{\xi}$ and $\left(A_{\xi}\right)_{\mathrm{Y}} \rightarrow\left(A_{\xi}^{R}\right)_{\mathrm{I}}=0,\left(A_{\eta}\right)_{\mathrm{I}} \rightarrow\left(A_{\eta}^{R}\right)_{\mathrm{I}}=0$. This argument also shows that the $\mathrm{R}$ gauge is a nonlocal gauge because due to the appearance of path-ordered exponentials in the solution of (4.10), the fields $h^{R}$ and $g^{R}$ are nonlocal functionals of the original field $g^{5}$. On the other hand, it is nearly a complete gauge because the conditions (4.1a) respectively (4.1b) and (4.9) only leave us with the freedom to perform global gauge transformations $g^{R} \rightarrow g^{R} h_{\circ}$ with space-time-independent group elements $h_{\circ} \in L^{\prime}$ respectively $h_{\circ} \in L$.

From our previous analysis, we now see that in the $\mathrm{R}$ gauge, the local gauge freedom has been completely eliminated, and with the help of a geometric identity, the field equation for the nonlinear $\sigma$-model has been reduced to a system of partial differential equations for certain fields $k_{\xi}^{R}=k_{\xi},\left(k_{\eta}^{R}\right)_{a}=\left(k_{\eta}\right)_{\mathrm{a}}$ (taking values in a) and $\phi^{R}=h^{R} \phi\left(h^{R}\right)^{-1}$ (taking values in $n$ ), namely:

$$
\begin{gathered}
\partial_{\eta} k_{\xi}=0, \\
\partial_{\eta}\left(\frac{1}{2}\left[\phi^{R},\left[k_{\xi}, \phi^{R}\right]\right]\right)_{\mathfrak{a}}=\partial_{\xi}\left(k_{\eta}\right)_{\mathfrak{a}}, \\
\square \phi^{R}=\left[\left(k_{\eta}\right)_{\mathfrak{a}},\left[k_{\xi}, \phi^{R}\right]\right]+\left[\partial_{\eta} \phi^{R},\left[k_{\xi}, \phi^{R}\right]\right]_{\mathfrak{n}} .
\end{gathered}
$$

5 All quantities referring to the reduction gauge will carry an upper index $\mathrm{R}$ 
This reduced system, which involves $\operatorname{dim} M+\operatorname{rank} M$ independent degrees of freedom (because $\operatorname{dim} \mathfrak{p}+2 \operatorname{dim} \mathfrak{a}=\operatorname{dim} M+\operatorname{rank} M$ ) and is globally $\operatorname{Ad}(L)$ invariant, can be viewed as a generalization of the well-known reduced systems [1, $13,14,18-20]$, at least for rank 1 spaces (such as the spheres $S^{N-1}$ and the complex projective spaces $\mathbb{C} P^{N-1}$ ), where in normalized coordinates, the number of independent degrees of freedom reduces to $\operatorname{dim} M-1$. Even there, however, we have not been able to find a Lagrangian from which to derive the system (4.11)-(4.13) by the usual variational principle, and hence promote it to the status of a genuine field theory. Actually, guided by the examples $M=S^{2}=\mathbb{C} P^{1}, M=S^{3}$, and $M=\mathbb{C} P^{2}[1,14]$, we believe that this is impossible, but that it becomes possible after performing an appropriate transformation of variables (which is yet to be found) and rewriting the reduced system as a system of partial differential equations in the new variables. In any case, it seems that much work remains to be done in this direction.

\section{Dual Symmetry and Local Conservation Laws}

The basis for all the known conservation laws - whether local or nonlocal - in nonlinear $\sigma$-models is the dual symmetry $[1,3,7]$. This is a transformation which associates with each solution $q=g H$ of the field equations an entire one-parameter family of solutions $q^{(\gamma)}=g^{(\gamma)} H$ of the field equations with the same action $S\left(q^{(\gamma)}\right)$ $=S(q)$, where $\gamma \in \mathbb{R}, \gamma \neq 0$, as follows $[3, \mathrm{I}]:$ Define $g^{(\gamma)}=U^{(\gamma)} g$, where the $G$-valued field $U^{(\gamma)}$ is the solution of the following compatible system of differential equations :

$$
\begin{gathered}
\partial_{\xi} U^{(\gamma)}=\left(1-\gamma^{-1}\right) U^{(\gamma)} j_{\xi} \\
\partial_{\eta} U^{(\gamma)}=(1-\gamma) U^{(\gamma)} j_{\eta}
\end{gathered}
$$

Then

$$
\begin{aligned}
& A_{\xi}^{(\gamma)}=A_{\xi}, \quad A_{\eta}^{(\gamma)}=A_{\eta}, \quad F_{\xi \eta}^{(\gamma)}=F_{\xi \eta}, \\
& k_{\xi}^{(\gamma)}=\gamma^{-1} k_{\xi}, \quad k_{\eta}^{(\gamma)}=\gamma k_{\eta}, \\
& j_{\xi}^{(\gamma)}=\gamma^{-1} U^{(\gamma)} j_{\xi} U^{(\gamma)^{-1}}, \quad j_{\eta}^{(\gamma)}=\gamma U^{(\gamma)} j_{\eta} U^{(\gamma)^{-1}},
\end{aligned}
$$

and the $G$-valued field $g^{(\gamma)}$ is the solution of the following compatible system of differential equations [to be compared with (3.14)]:

$$
\begin{gathered}
\partial_{\xi} g^{(\gamma)}=g^{(\gamma)}\left(A_{\xi}+\gamma^{-1} k_{\xi}\right), \\
\partial_{\eta} g^{(\gamma)}=g^{(\gamma)}\left(A_{\eta}+\gamma k_{\eta}\right) .
\end{gathered}
$$

From the corresponding transformation laws for the $j_{\mu}, A_{\mu}$, and $k_{\mu}$, it follows that under gauge transformations $g \rightarrow g h, U^{(\gamma)}$ is invariant and $g^{(\gamma)}$ is covariant (i.e. $U^{(\gamma)} \rightarrow U^{(\gamma)}$ and $\left.g^{(\gamma)} \rightarrow g^{(\gamma)} h\right)$, and both are uniquely determined up to multiplication from the left by a space-time-independent, but (in general) $\gamma$-dependent normalization factor $U_{\circ}^{(\gamma)} \in G$. For convenience, we choose a reference point $x_{\circ}$ in spacetime around which the given solution $q=g H$ - and hence any of the transformed solutions $q^{(\gamma)}=g^{(\gamma)} H$ - is regular in the sense described at the beginning of Sect. 4 
(i.e. around which the fields $k_{\mu}$ - and hence any of the transformed fields $k_{\mu}^{(\gamma)}$ - take values in the regular set $m_{r}^{\circ}$ in $m$ ), and put $U_{\circ}^{(\gamma)}=U^{(\gamma)}\left(x_{\circ}\right)$. Then we relate the values of this normalization factor for $\gamma>0$ and for $\gamma<0$ by requiring [3, II]

$$
U^{(-\gamma)}=\sigma\left(U^{(\gamma)}\right) Q \quad \text { or equivalently } \quad g^{(-\gamma)}=\sigma\left(g^{(\gamma)}\right) ;
$$

note that due to the differential equations (5.1), (5.3), this condition holds everywhere if it holds at $x_{\circ}$. Moreover, we require that for $\gamma$ near 1 ,

$$
U^{(\gamma)}\left(x_{\circ}\right)=1 \text { or equivalently } g^{(\gamma)}\left(x_{\circ}\right)=g\left(x_{\circ}\right),
$$

and for $\gamma$ near 0 as well as for $\gamma$ near $\infty$

$$
U^{(\gamma)}\left(x_{\circ}\right)=g\left(x_{\circ}\right)^{-1} \quad \text { or equivalently } \quad g^{(\gamma)}\left(x_{\circ}\right)=1 .
$$

Now on the hand, the nonlocal conservation laws are known [3, I, 7] to arise from a series expansion of the gauge invariant, $\gamma$-dependent conservation law

$$
\partial_{\xi} j_{\eta}^{(\gamma)}+\partial_{\eta} j_{\xi}^{(\gamma)}=0
$$

around the point $\gamma=1$ [in powers of $w=(\gamma-1) /(\gamma+1)$ around $w=0$ ]; moreover, the normalization condition (5.5) guarantees that for $\gamma$ near $1, j_{\mu}^{(\gamma)}[\mathrm{cf} .(5.2)]$ is covariant under global symmetry transformations $q \rightarrow g_{\circ} q, g \rightarrow g_{\circ} g$ with space-timeindependent group elements $g_{\circ} \in G$. On the other hand, the known examples $[1,8-13]$ suggest that two series of local conservation laws should arise from an asymptotic series expansion of some similar gauge invariant, $\gamma$-dependent conservation law

$$
\partial_{\xi} J_{\eta}^{(\gamma)}+\partial_{\eta} J_{\xi}^{(\gamma)}=0
$$

around the point $\gamma=0$ (in powers of $\gamma$ around $\gamma=0$ ) and around the point $\gamma=\infty$ (in powers of $\gamma^{-1}$ around $\gamma^{-1}=0$ ); moreover, the normalization condition (5.6) should guarantee that for $\gamma$ near 0 and for $\gamma$ near $\infty, J_{\mu}^{(\gamma)}$ is invariant under global symmetry transformations $q \rightarrow g_{\circ} q, g \rightarrow g_{\circ} g$ with space-time-independent group elements $g_{\circ} \in G$. The two series should then correspond to each other under the usual interchange $\gamma \leftrightarrow \gamma^{-1}, \xi \leftrightarrow \eta$, so that without loss of generality, we may (and shall) concentrate on the first of them (obtained by expanding around $\gamma=0$ ).

In the following, we want to show that all this is indeed the case, and in fact, it turns out that

$$
J_{\xi}^{(\gamma)}=+\partial_{\xi} a^{(\gamma)}, \quad J_{\eta}^{(\gamma)}=-\partial_{\eta} a^{(\gamma)},
$$

where the field $a^{(\gamma)}$ is the "horizontal abelian part" (a-component) in a decomposition

$$
g^{(\gamma)}=\exp \left(a^{(\gamma)}\right) h^{R} \exp \left(l^{(\gamma)}\right) \exp \left(\omega^{(\gamma)}\right)
$$

of the field $g^{(\gamma)}$ which is obtained as follows:

First, since we are interested in an expansion around $\gamma=0$, the leading singularity in the system (5.3) of differential equations for the field $g^{(\gamma)}$ comes from the $\gamma^{-1} k_{\xi}$ term, and it seems reasonable to simplify this term as much as possible by going to the PR gauge. More precisely, we assume that on the domain of spacetime under consideration, the original solution $q=g H$ of the field equations is 
regular in the sense explained at the beginning of Sect. 4, and we impose the PR gauge condition (4.1); then $\operatorname{ad}\left(k_{\xi}\right)$ - considered as a field of linear transformations from $\mathfrak{n}$ to $\mathfrak{p}$ and/or from $\mathfrak{p}$ to $\mathfrak{n}$ - takes values in the invertible linear transformations because $\lambda\left(k_{\xi}\right) \neq 0$ for all restricted roots $\lambda \in \Sigma$. Next, we define $h^{R}$ to be the unique $L$-valued field which satisfies (4.10) and the normalization condition

$$
h^{R}\left(x_{\circ}\right)=1
$$

thus in particular, $g \rightarrow g^{R}=g\left(h^{R}\right)^{-1}$ is the residual gauge transformation leading from the PR gauge to the $\mathrm{R}$ gauge. (For more details, we refer to Sect. 4.) Now using the fact that the map

$$
\begin{gathered}
\mathfrak{g}=\mathfrak{a} \oplus \mathbb{l} \oplus(\mathfrak{n} \oplus \mathfrak{p}) \rightarrow G \\
(a, \tilde{l}, \tilde{\omega}) \mapsto \exp (a) \exp (\tilde{l}) \exp (\tilde{\omega})
\end{gathered}
$$

is a diffeomorphism from an open neighbourhood of 0 in $\mathfrak{g}$ onto an open neighbourhood $W$ of 1 in $G$, we may assume without loss of generality that on the domain of space-time under consideration, there exist uniquely determined fields $a^{(\gamma)}, \tilde{l}^{(\gamma)}$, and $\tilde{\omega}^{(\gamma)}$ taking values in $\mathfrak{a}, \mathfrak{l}$, and $\mathfrak{n} \oplus \mathfrak{p}$, respectively, such that

$$
g^{(\gamma)}\left(h^{R}\right)^{-1}=\exp \left(a^{(\gamma)}\right) \exp \left(\tilde{l}^{(\gamma)}\right) \exp \left(\tilde{\omega}^{(\gamma)}\right)
$$

[Indeed, $W$ being an open neighbourhood of 1 in $G$, the points $x$ satisfying $g^{(\gamma)}(x) h^{R}(x)^{-1} \in W$ for $\gamma$ near 0 constitute a domain - i.e. an open subset - in spacetime which, according to the normalization conditions (5.6) and (5.11), contains our reference point $x_{\circ}$, and our assumption simply means that in addition to our previous regularity condition, we focus attention on this domain only.] Finally, we define fields $a^{(\gamma)}, l^{(\gamma)}$, and $\omega^{(\gamma)}$ taking values in $\mathfrak{a}, \mathfrak{l}$, and $n \oplus \mathfrak{p}$, respectively, by setting

$$
\begin{gathered}
l^{(\gamma)}=\operatorname{Ad}\left(h^{R}\right)^{-1} \tilde{l}^{(\gamma)}=\left(h^{R}\right)^{-1} \tilde{l}^{(\gamma)} h^{R}, \\
\omega^{(\gamma)}=\operatorname{Ad}\left(h^{R}\right)^{-1} \tilde{\omega}^{(\gamma)}=\left(h^{R}\right)^{-1} \tilde{\omega}^{(\gamma)} h^{R} .
\end{gathered}
$$

From the corresponding transformation laws for the $g^{(\gamma)}$ and for $h^{R}$ [cf. (4.10)], and from the $\operatorname{Ad}(L)$-invariance of the direct decomposition $\mathfrak{g}=\mathfrak{a} \oplus \mathfrak{l} \oplus(\mathfrak{n} \oplus \mathfrak{p})$ [cf. (2.11)], it follows that under residual gauge transformations $g \rightarrow g h, a^{(\gamma)}, \tilde{l}^{(\gamma)}, \tilde{\omega}^{(\gamma)}$ are invariant and $l^{(\gamma)}, \omega^{(\gamma)}$ are covariant (i.e. $a^{(\gamma)} \rightarrow a^{(\gamma)}, \tilde{l}^{(\gamma)} \rightarrow \tilde{l}^{(\gamma)}, \tilde{\omega}^{(\gamma)} \rightarrow \tilde{\omega}^{(\gamma)}$ and $\left.l^{(\gamma)} \rightarrow h^{-1} l^{(\gamma)} h, \omega^{(\gamma)} \rightarrow h^{-1} \omega^{(\gamma)} h\right)$, which motivates the introduction of an $L$-covariant derivative for the latter:

$$
\begin{gathered}
D_{\mu} l^{(\gamma)}=\partial_{\mu} l^{(\gamma)}+\left[\left(A_{\mu}\right)_{\mathfrak{I}} l^{(\gamma)}\right], \\
D_{\mu} \omega^{(\gamma)}=\partial_{\mu} \omega^{(\gamma)}+\left[\left(A_{\mu}\right)_{\mathfrak{I}}, \omega^{(\gamma)}\right] .
\end{gathered}
$$

Moreover, the normalization conditions (5.6) and (5.11) guarantee that for $\gamma$ near 0 , all the fields $g^{(\gamma)}, a^{(\gamma)}, \tilde{l}^{(\gamma)}, \tilde{\omega}^{(\gamma)}, h^{R}, l^{(\gamma)}, \omega^{(\gamma)}$ are invariant under global symmetry transformations $q \rightarrow g_{\circ} q, g \rightarrow g_{\circ} g$ with space-time-independent group elements $g_{\circ} \in G$, while the normalization condition (5.4) is equivalent to

$$
\begin{array}{lll}
a^{(-\gamma)}=-a^{(\gamma)}, & \tilde{l}^{(-\gamma)}=\tilde{l}^{(\gamma)}, & \tilde{\omega}^{(-\gamma)}=\dot{\sigma}\left(\tilde{\omega}^{(\gamma)}\right), \\
& l^{(-\gamma)}=l^{(\gamma)}, & \omega^{(-\gamma)}=\dot{\sigma}\left(\omega^{(\gamma)}\right) .
\end{array}
$$


Our next step is to insert the decomposition (5.10) into the system (5.3) of differential equations and to isolate the term containing the derivatives of $a^{(\gamma)}$. To this end, we compute

$$
\begin{aligned}
- & \partial_{\mu}\left(\exp \left(\omega^{(\gamma)}\right)\right) \exp \left(-\omega^{(\gamma)}\right) \\
= & -\partial_{\mu}\left(\exp \left(-l^{(\gamma)}\right)\left(h^{R}\right)^{-1} \exp \left(-a^{(\gamma)}\right) g^{(\gamma)}\right) g^{(\gamma)-1} \exp \left(a^{(\gamma)}\right) h^{R} \exp \left(l^{(\gamma)}\right) \\
= & -\partial_{\mu}\left(\exp \left(-l^{(\gamma)}\right)\right) \exp \left(l^{(\gamma)}\right)+\exp \left(-l^{(\gamma)}\right)\left(h^{R}\right)^{-1} \partial_{\mu} h^{R} \exp \left(l^{(\gamma)}\right) \\
& +\partial_{\mu} a^{(\gamma)}-\exp \left(\omega^{(\gamma)}\right) g^{(\gamma)-1} \partial_{\mu} g^{(\gamma)} \exp \left(-\omega^{(\gamma)}\right)
\end{aligned}
$$

and apply the formula for the derivative of the exponential map (2.7) [15, p. 105], which - when combined with the chain rule - states that for any $g$-valued field $X$,

$$
\begin{aligned}
& \exp (-X) \partial_{\mu}(\exp (X))=+\frac{1-\exp (-\operatorname{ad}(X))}{\operatorname{ad}(X)} \partial_{\mu} X=\sum_{k=0}^{\infty} \frac{(-1)^{k}}{(k+1) !}[\underbrace{\left.X, \ldots,\left[X, \partial_{\mu} X\right] \ldots\right]}_{k \text { factors }} \\
& \left.\partial_{\mu}(\exp (X)) \exp (-X)=-\frac{1-\exp (+\operatorname{ad}(X))}{\operatorname{ad}(X)} \partial_{\mu} X=\sum_{k=0}^{\infty} \frac{1}{(k+1) !}[\overbrace{X, \ldots,[X}, \partial_{\mu} X] \ldots\right] .
\end{aligned}
$$

Using (4.10) and (5.2), (5.3), this gives

$$
\begin{aligned}
\partial_{\mu} a^{(\gamma)}= & -\frac{1-\exp \left(-\operatorname{ad}\left(l^{(\gamma)}\right)\right)}{\operatorname{ad}\left(l^{(\gamma)}\right)} \partial_{\mu} l^{(\gamma)}+\exp \left(-\operatorname{ad}\left(l^{(\gamma)}\right)\right)\left(A_{\mu}\right)_{\Gamma} \\
& +\frac{1-\exp \left(+\operatorname{ad}\left(\omega^{(\gamma)}\right)\right)}{\operatorname{ad}\left(\omega^{(\gamma)}\right)} \partial_{\mu} \omega^{(\gamma)}+\exp \left(\operatorname{ad}\left(\omega^{(\gamma)}\right)\right)\left(A_{\mu}+k_{\mu}^{(\gamma)}\right),
\end{aligned}
$$

i.e.

$$
\begin{aligned}
\partial_{\mu} a^{(\gamma)}= & -\frac{1-\exp \left(-\operatorname{ad}\left(l^{(\gamma)}\right)\right)}{\operatorname{ad}\left(l^{(\gamma)}\right)} D_{\mu} l^{(\gamma)}+\frac{1-\exp \left(+\operatorname{ad}\left(\omega^{(\gamma)}\right)\right)}{\operatorname{ad}\left(\omega^{(\gamma)}\right)} D_{\mu} \omega^{(\gamma)} \\
& +\exp \left(\operatorname{ad}\left(\omega^{(\gamma)}\right)\right)\left(\left(A_{\mu}\right)_{\mathfrak{p}}+k_{\mu}^{(\gamma)}\right),
\end{aligned}
$$

or writing out the power series,

$$
\begin{aligned}
\partial_{\mu} a^{(\gamma)}=\sum_{k=0}^{\infty}\{ & -\frac{(-1)^{k}}{(k+1) !}\left[l^{(\gamma)}, \ldots,\left[l^{(\gamma)}, D_{\mu} l^{(\gamma)}\right] \ldots\right]-\frac{1}{(k+1) !}\left[\omega^{(\gamma)}, \ldots,\left[\omega^{(\gamma)}, D_{\mu} \omega^{(\gamma)}\right] \ldots\right] \\
& \left.+\frac{1}{k !}\left[\omega^{(\gamma)}, \ldots,\left[\omega^{(\gamma)},\left(A_{\mu}\right)_{\mathfrak{p}}+k_{\mu}^{(\gamma)}\right] \ldots\right]\right\} .
\end{aligned}
$$

One of the main motivations for performing the decomposition (5.10), with the given ordering of the factors, comes from the observation that in this way, the singular behaviour of the fields $g^{(\gamma)}$ near $\gamma=0$ - which is already obvious from (5.3) - becomes completely concentrated in the a-component, where it just appears as a simple pole, and in the l-component, where it is irrelevant because it can in principle be gauged away via a $\gamma$-dependent residual gauge transformation; moreover, as a reflection of the fact that we are working in the PR gauge, it turns out that the other components vanish at $\gamma=0$. These properties are formally expressed by disregarding the l-component of (5.10) and making a power series 
expansion Ansatz of the form

$$
a^{(\gamma)}=\sum_{n=-1}^{\infty} \gamma^{n} a^{(n)}, \quad \omega^{(\gamma)}=\sum_{n=+1}^{\infty} \gamma^{n} \omega^{(n)}
$$

for the other components, where in order to satisfy (5.16),

$$
a^{(n)}=0 \text { for } n \text { even, } \begin{aligned}
& \omega^{(n)} \text { takes values in } \mathfrak{p} \text { for } n \text { even, } \\
& \omega^{(n)} \text { takes values in } n \text { for } n \text { odd }
\end{aligned}
$$

From the corresponding transformation laws for the $a^{(\gamma)}$ and $\omega^{(\gamma)}$, it follows that under residual gauge transformations $g \rightarrow g h$, the $a^{(n)}$ are invariant and the $\omega^{(n)}$ are covariant (i.e. $a^{(n)} \rightarrow a^{(n)}$ and $\left.\omega^{(n)} \rightarrow h^{-1} \omega^{(n)} h\right)$, which motivates the introduction of an $L$-covariant derivative for the latter:

$$
D_{\mu} \omega^{(n)}=\partial_{\mu} \omega^{(n)}+\left[\left(A_{\mu}\right)_{\mathrm{l}}, \omega^{(n)}\right] .
$$

Moreover, all the coefficient fields $a^{(n)}, \omega^{(n)}$ are invariant under global symmetry transformations $q \rightarrow g_{\circ} q, g \rightarrow g_{\circ} g$ with space-time-independent group elements $g_{\circ} \in G$.

Inserting (5.19) and (5.2) into (5.18), discarding the l-component, and collecting the coefficients of the various powers of $\gamma$, we obtain the following two series of equations [where we have also exploited (4.4) in order to eliminate $\left(A_{\eta}\right)_{\mathfrak{p}}$ ]:

\section{$\xi$-Series}

$$
\begin{aligned}
\partial_{\xi} a^{(-1)} \underset{\bmod }{=} & k_{\xi} \\
\partial_{\xi} a^{(0)} \underset{\bmod I}{=} & \left(A_{\xi}\right)_{\mathfrak{p}}+\left[\omega^{(1)}, k_{\xi}\right] \\
\partial_{\xi} a^{(1)} \underset{\operatorname{modr}}{=} & -D_{\xi} \omega^{(1)}+\left[\omega^{(1)},\left(A_{\xi}\right)_{\mathfrak{p}}\right]+\left[\omega^{(2)}, k_{\xi}\right]+\frac{1}{2}\left[\omega^{(1)},\left[\omega^{(1)}, k_{\xi}\right]\right] \\
\partial_{\xi} a^{(2)} \underset{\bmod I}{=} & -D_{\xi} \omega^{(2)}-\frac{1}{2}\left[\omega^{(1)}, D_{\xi} \omega^{(1)}\right]+\left[\omega^{(2)},\left(A_{\xi}\right)_{\mathfrak{p}}\right]+\frac{1}{2}\left[\omega^{(1)},\left[\omega^{(1)},\left(A_{\xi}\right)_{\mathfrak{p}}\right]\right] \\
& +\left[\omega^{(3)}, k_{\xi}\right]+\frac{1}{2}\left[\omega^{(2)},\left[\omega^{(1)}, k_{\xi}\right]\right]+\frac{1}{2}\left[\omega^{(1)},\left[\omega^{(2)}, k_{\xi}\right]\right] \\
& +\frac{1}{6}\left[\omega^{(1)},\left[\omega^{(1)},\left[\omega^{(1)}, k_{\xi}\right]\right]\right]
\end{aligned}
$$

etc., and for general $n \geqq 2$,

$$
\begin{gathered}
\partial_{\xi} a^{(n)}=-D_{\xi} \omega^{(n)}-\sum_{k=1}^{n-1} \sum_{\substack{n_{1}, \ldots, n_{k}, l=1 \\
n_{1}+\ldots+n_{k}+l=n}}^{n-1} \frac{1}{(k+1) !}\left[\omega^{\left(n_{1}\right)}, \ldots,\left[\omega^{\left(n_{k}\right)}, D_{\xi} \omega^{(l)}\right] \ldots\right] \\
+\sum_{k=1}^{n} \sum_{\substack{n_{1}, \ldots, n_{k}=1 \\
n_{1}+\ldots+n_{k}=n}}^{n} \frac{1}{k !}\left[\omega^{\left(n_{1}\right)}, \ldots,\left[\omega^{\left(n_{k}\right)},\left(A_{\xi}\right)_{\mathfrak{p}}\right] \ldots\right] \\
+\left[\omega^{(n+1)}, k_{\xi}\right]+\sum_{k=1}^{n} \sum_{\substack{n_{1}, \ldots, n_{k}, l=1 \\
n_{1}+\ldots+n_{k}+l=n+1}}^{n} \frac{1}{(k+1) !}\left[\omega^{\left(n_{1}\right)}, \ldots,\left[\omega^{\left(n_{k}\right)},\left[\omega^{(l)}, k_{\xi}\right]\right] \ldots\right] .
\end{gathered}
$$


$\eta$-Series

$$
\begin{aligned}
& \partial_{\eta} a^{(-1)} \underset{\text { modr }}{=} 0, \\
& \partial_{\eta} a^{(0)} \underset{\text { modI }}{=} 0, \\
& \partial_{\eta} a^{(1)} \underset{\bmod I}{=}-D_{\eta} \omega^{(1)}+k_{\eta}, \\
& \partial_{\eta} a^{(2)} \underset{\bmod I}{=}-D_{\eta} \omega^{(2)}-\frac{1}{2}\left[\omega^{(1)}, D_{\eta} \omega^{(1)}\right]+\left[\omega^{(1)}, k_{\eta}\right],
\end{aligned}
$$

etc., and for general $n \geqq 2$,

$$
\begin{aligned}
\partial_{\eta} a^{(n)}= & -D_{\eta} \omega^{(n)}-\sum_{k=1}^{n-1} \sum_{\substack{n_{1}, \ldots, n_{k}, l=1 \\
n_{1}+\ldots+n_{k}+l=n}}^{n-1} \frac{1}{(k+1) !}\left[\omega^{\left(n_{1}\right)}, \ldots,\left[\omega^{\left(n_{k}\right)}, D_{\eta} \omega^{(l)}\right] \ldots\right] \\
& +\sum_{k=1}^{n-1} \sum_{\substack{n_{1}, \ldots, n_{k}=1 \\
n_{1}+\ldots+n_{k}=n-1}}^{n-1} \frac{1}{k !}\left[\omega^{\left(n_{1}\right)}, \ldots,\left[\omega^{\left(n_{k}\right)}, k_{\eta}\right] \ldots\right] .
\end{aligned}
$$

As we shall see, this system of equations can be solved recursively: We start from the observation that (5.22) and (5.24) constitute a system of differential equations for the field $a^{(-1)}$ whose compatibility condition is the zeroth local conservation law (4.6), while (5.23.0) and (5.25.0), together with the restriction imposed by (5.20), are solved by

$$
\omega^{(1)}=\operatorname{ad}\left(k_{\xi}\right)^{-1}\left(A_{\xi}\right)_{p}
$$

[cf. (4.2)]. More generally, the subsidiary condition (5.20) implies ${ }^{6}$ that all terms appearing in the $n^{\text {th }}$ equation (in both series) belong to $\mathfrak{m}$ respectively to $\mathfrak{h}$ if $n$ is odd respectively even. Thus splitting this equation into its a-component and its $n$-component if $n$ is odd, and into its l-component (which is trivial) and its $\mathfrak{p}$-component if $n$ is even, we obtain the following series of equations [where we have also exploited (5.26) in order to eliminate $\left(A_{\xi}\right)_{\mathfrak{p}}$ in favor of $\left.\omega^{(1)}\right]$ :

$\xi$-Series

$$
\begin{aligned}
\partial_{\xi} a^{(1)}= & -\frac{1}{2}\left[\omega^{(1)},\left[\omega^{(1)}, k_{\xi}\right]\right]_{\mathfrak{a}}, \\
\omega^{(2)}= & \operatorname{ad}\left(k_{\xi}\right)^{-1}\left\{-D_{\xi} \omega^{(1)}-\frac{1}{2}\left[\omega^{(1)},\left[\omega^{(1)}, k_{\xi}\right]\right]_{\mathfrak{n}}\right\}, \\
\omega^{(3)}= & \operatorname{ad}\left(k_{\xi}\right)^{-1}\left\{-D_{\xi} \omega^{(2)}-\frac{1}{2}\left[\omega^{(1)}, D_{\xi} \omega^{(1)}\right]_{\mathfrak{p}}\right. \\
& -\frac{1}{2}\left[\omega^{(2)},\left[\omega^{(1)}, k_{\xi}\right]\right]_{\mathfrak{p}}+\frac{1}{2}\left[\omega^{(1)},\left[\omega^{(2)}, k_{\xi}\right]\right]_{\mathfrak{p}} \\
& \left.-\frac{1}{3}\left[\omega^{(1)},\left[\omega^{(1)},\left[\omega^{(1)}, k_{\xi}\right]\right]\right]_{\mathfrak{p}}\right\}
\end{aligned}
$$

6 Here, we use the fact [easily proved from (2.5) by induction on $k$ ] that for integers $n_{1}, \ldots, n_{k}, n_{k+1}$ and elements $X_{1}^{\left(n_{1}\right)}, \ldots, X_{k}^{\left(n_{k}\right)}, X_{k+1}^{\left(n_{k+1}\right)}$ of $\mathfrak{g}$ with $X_{i}^{\left(n_{i}\right)} \in \mathfrak{m}$ respectively $\mathfrak{h}$ for $n_{i}$ odd respectively even, we have $\left[X_{1}^{\left(n_{1}\right)}, \ldots,\left[X_{k}^{\left(n_{k}\right)}, X_{k+1}^{\left(n_{k+1}\right)}\right] \ldots\right] \in \mathfrak{m}$ respectively $\mathfrak{h}$ for $n_{1}+\ldots+n_{k}+n_{k+1}$ odd respectively even 
etc., and for general $n \geqq 2$,

$$
\begin{aligned}
& \partial_{\xi} a^{(n)}=-\sum_{k=1}^{n-1} \sum_{\substack{n_{1}, \ldots, n_{k}, l=1 \\
n_{1}+\ldots+n_{k}+l=n}}^{n-1} \frac{1}{(k+1) !}\left[\omega^{\left(n_{1}\right)}, \ldots,\left[\omega^{\left(n_{k}\right)}, D_{\xi} \omega^{(l)}\right] \ldots\right]_{\mathfrak{a}} \\
& +\sum_{k=1}^{n} \sum_{\substack{n_{1}, \ldots, n_{k}, l=1 \\
n_{1}+\ldots+n_{k}+l=n+1}}^{n} \frac{c_{k, l}}{(k+1) !}\left[\omega^{\left(n_{1}\right)}, \ldots,\left[\omega^{\left(n_{k}\right)},\left[\omega^{(l)}, k_{\xi}\right]\right] \ldots\right]_{\mathfrak{a}}, \quad(5.27 . n)_{\mathfrak{a}} \\
& \omega^{(n+1)}=\operatorname{ad}\left(k_{\xi}\right)^{-1}\left\{-D_{\xi} \omega^{(n)}-\sum_{k=1}^{n-1} \sum_{\substack{n_{1}, \ldots, n_{k}, l=1 \\
n_{1}+\ldots+n_{k}+l=n}}^{n-1} \frac{1}{(k+1) !}\left[\omega^{\left(n_{1}\right)}, \ldots,\left[\omega^{\left(n_{k}\right)}, D_{\xi} \omega^{(l)}\right] \ldots\right]_{\mathfrak{n}}\right. \\
& \left.+\sum_{k=1}^{n} \sum_{\substack{n_{1}, \ldots, n_{k}, l=1 \\
n_{1}+\ldots+n_{k}+l=n+1}}^{n} \frac{c_{k, l}}{(k+1) !}\left[\omega^{\left(n_{1}\right)}, \ldots,\left[\omega^{\left(n_{k}\right)},\left[\omega^{(l)}, k_{\xi}\right]\right] \ldots\right]_{\mathfrak{n}}\right\} \quad(5.27 . n)_{\mathfrak{n}}
\end{aligned}
$$

if $n$ is odd, and

$$
\begin{aligned}
\omega^{(n+1)}= & \operatorname{ad}\left(k_{\xi}\right)^{-1}\left\{-D_{\xi} \omega^{(n)}-\sum_{k=1}^{n-1} \sum_{\substack{n_{1}, \ldots, n_{k}, l=1 \\
n_{1}+\ldots+n_{k}+l=n}}^{n-1} \frac{1}{(k+1) !}\left[\omega^{\left(n_{1}\right)}, \ldots,\left[\omega^{\left(n_{k}\right)}, D_{\xi} \omega^{(l)}\right] \ldots\right]_{\mathfrak{p}}\right. \\
& \left.+\sum_{k=1}^{n} \sum_{\substack{n_{1}, \ldots, n_{k}, l=1 \\
n_{1}+\ldots+n_{k}+l=n+1}}^{n} \frac{c_{k, l}}{(k+1) !}\left[\omega^{\left(n_{1}\right)}, \ldots,\left[\omega^{\left(n_{k}\right)},\left[\omega^{(l)}, k_{\xi}\right]\right] \ldots\right]_{\mathfrak{p}}\right\} \quad(5.27 . n)_{\mathfrak{p}}
\end{aligned}
$$

if $n$ is even, where

$$
c_{k, 1}=-k, \quad c_{k, l}=1 \quad \text { for } \quad l \geqq 2 .
$$

$\eta$-Series

$$
\begin{aligned}
\partial_{\eta} a^{(1)} & =\left(k_{\eta}\right)_{\mathfrak{a}}, \\
D_{\eta} \omega^{(1)} & =\left(k_{\eta}\right)_{\mathfrak{n}} \\
D_{\eta} \omega^{(2)} & =-\frac{1}{2}\left[\omega^{(1)}, D_{\eta} \omega^{(1)}\right]_{\mathfrak{p}}+\left[\omega^{(1)}, k_{\eta}\right]_{\mathfrak{p}}
\end{aligned}
$$

etc., and for general $n \geqq 2$,

$$
\begin{aligned}
& \partial_{\eta} a^{(n)}=-\sum_{k=1}^{n-1} \sum_{\substack{n_{1}, \ldots, n_{k}, l=1 \\
n_{1}+\ldots+n_{k}+l=n}}^{n-1} \frac{1}{(k+1) !}\left[\omega^{\left(n_{1}\right)}, \ldots,\left[\omega^{\left(n_{k}\right)}, D_{\eta} \omega^{(l)}\right] \ldots\right]_{a} \\
& +\sum_{k=1}^{n-1} \sum_{\substack{n_{1}, \ldots, n_{k}=1 \\
n_{1}+\ldots+n_{k}=n-1}}^{n-1} \frac{1}{k !}\left[\omega^{\left(n_{1}\right)}, \ldots,\left[\omega^{\left(n_{k}\right)}, k_{\eta}\right] \ldots\right]_{a} \\
& D_{\eta} \omega^{(n)}=-\sum_{k=1}^{n-1} \sum_{\substack{n_{1}, \ldots, n_{k}, l=1 \\
n_{1}+\ldots+n_{k}+l=n}}^{n-1} \frac{1}{(k+1) !}\left[\omega^{\left(n_{1}\right)}, \ldots,\left[\omega^{\left(n_{k}\right)}, D_{\eta} \omega^{(l)}\right] \ldots\right]_{\mathfrak{n}} \\
& +\sum_{k=1}^{n-1} \sum_{\substack{n_{1}, \ldots, n_{k}=1 \\
n_{1}+\ldots+n_{k}=n-1}}^{n-1} \frac{1}{k !}\left[\omega^{\left(n_{1}\right)}, \ldots,\left[\omega^{\left(n_{k}\right)}, k_{\eta}\right] \ldots\right]_{\mathfrak{n}}
\end{aligned}
$$


if $n$ is odd, and

$$
\begin{aligned}
D_{\eta} \omega^{(n)=} & -\sum_{k=1}^{n-1} \sum_{\substack{n_{1}, \ldots, n_{k}, l=1 \\
n_{1}+\ldots+n_{k}+l=n}}^{n-1} \frac{1}{(k+1) !}\left[\omega^{\left(n_{1}\right)}, \ldots,\left[\omega^{\left(n_{k}\right)}, D_{\eta} \omega^{(l)}\right] \ldots\right]_{\mathfrak{p}} \\
& +\sum_{k=1}^{n-1} \sum_{\substack{n_{1}, \ldots, n_{k}=1 \\
n_{1}+\ldots+n_{k}=n-1}}^{n-1} \frac{1}{k !}\left[\omega^{\left(n_{1}\right)}, \ldots,\left[\omega^{\left(n_{k}\right)}, k_{\eta}\right] \ldots\right]_{\mathfrak{p}}
\end{aligned}
$$

if $n$ is even.

It is now obvious that the $\xi$-series of Eqs. (5.27), together with the subsidiary condition (5.20), can be satisfied identically if we simply define $\partial_{\xi} a^{(n)}$ (in terms of $\left.\omega^{(1)}, \ldots, \omega^{(n)}\right)$ and $\omega^{(n)}$ (in terms of $\omega^{(1)}, \ldots, \omega^{(n-1)}$ ) by repeated application of (5.27). Moreover, we see (by induction on $n$ ) that $\partial_{\xi} a^{(n)}$ and $\omega^{(n)}$ are linear combinations of terms which result from repeatedly applying any of the following four operations, in any possible order, to $\left(A_{\xi}\right)_{\mathfrak{p}}$ :

a) The $L$-covariant $\xi$-derivative $D_{\xi}$.

b) The transformation $\operatorname{ad}\left(k_{\xi}\right)^{-1}$.

c) The commutator $[\cdot, \cdot]$.

d) The projection to $\mathfrak{a}, \mathfrak{n}, \mathfrak{l}$ or $\mathfrak{p}$.

Of course, there are various additional restrictions: For example, the last projection (to be performed after having taken all commutators) has to map onto the right subspace (e.g. never onto l), and the total number of $\xi$-derivatives never exceeds $n-1$. However, we have not been able to find a closed expression for these terms. Finally, we do except the $\eta$-series of Eq. (5.29) to be satisfied as a consequence of the field equations, but lacking the aforementioned explicit expression, we have no way of checking this directly (except in the lowest few orders ${ }^{7}$ ), and lacking an independent justification for the validity of our Ansatz (5.19), (5.20), we have no a priori guarantee that this is so, either. [To illustrate this last point, note that if we had also made a power series expansion Ansatz of the form

$$
l^{(\gamma)}=\sum_{n=0}^{\infty} \gamma^{n} l^{(n)}
$$

for the I-component, where in order to satisfy (5.16),

$$
l^{(n)}=0 \text { for } n \text { odd, }
$$

we would have obtained $l^{(0)}=0$, but the equations for $D_{\xi} l^{(2)}$ and $D_{\eta} l^{(2)}$ would not be compatible, thus leading us to a contradiction.] Thus there is still a gap in our derivation of the $r^{\text {th }}$ local conservation law - which is simply the compatibility condition

$$
\partial_{\eta} \partial_{\xi} a^{(n)}=\partial_{\xi} \partial_{\eta} a^{(n)}
$$

7 We have, however, checked $(5.29 .1)_{\mathfrak{n}},(5.29 .2)_{\mathfrak{p}},(5.29 .3)_{\mathfrak{n}}$ and also $(5.29 .1)_{\mathfrak{a}}-$ the latter by proving it to be compatible with $(5.27 .1)_{a}$; cf. (4.7) 
for the Eqs. (5.27.n $)_{a}$ and (5.29.n), with $n=2 r-1-$ for general $r, r \geqq 2,{ }^{7}$ and this is why we have deliberately avoided the usual terminology of " infinite series" of local conservation laws".

We want to emphasize, however, that this gap is common to all the various existing methods of deriving local conservation laws for nonlinear $\sigma$-models $[1,8-13]$, and that our method has the advantage of displaying at least the first higher local conservation law for the nonlinear $\sigma$-model on an arbitrary Riemannian symmetric space in an amazingly simple and explicit form, namely [cf. (4.2), (4.7), (5.26), (5.27.1) $\left.\mathfrak{a},(5.29 .1)_{\mathfrak{a}},(5.30)\right]$ :

$$
\partial_{\eta}\left(\frac{1}{2}\left[\operatorname{ad}\left(k_{\xi}\right)^{-1}\left(A_{\xi}\right)_{\mathfrak{p}},\left(A_{\xi}\right)_{\mathfrak{p}}\right]\right)_{\mathfrak{a}}=\partial_{\xi}\left(k_{\eta}\right)_{\mathfrak{a}}
$$

\section{Examples}

In this last section, we want to give examples which illustrate the general concepts and constructions of Sects. 2-5, and we shall concentrate on the real Grassmann manifolds $S O(N) / S O(p) \times S O(q)$ and the complex Grassmann manifolds $S U(N) / S(U(p) \times U(q))$, where $N=p+q$ and $p \leqq q$. First, we write down explicitly the basic decomposition (2.11) of the corresponding Lie algebra and exhibit the first higher local conservation law (5.31) in a different form. As before, this conservation law is manifestly invariant under residual gauge transformations, but in some cases, it can be restated in a form which is manifestly invariant even under general gauge transformations: Apart from the rank 1 spaces, i.e. the spheres $S^{N-1}=S O(N) / S O(N-1)$ and the complex projective spaces $\mathbb{C} P^{N-1}$ $=S U(N) / S(U(1) \times U(N-1))$, where our conservation law reduces to the usual one $[1,8]$, this applies to the real rank 2 Grassmannians $S O(N) / S O(2) \times S O(N-2)$ as well as to the complex rank2 Grassmannians $S U(N) / S(U(2) \times U(N-2))$. Here, algebraic simplifications occur because for a positive definite hermitean $(2 \times 2)$ matrix, one can explicitly write down two linearly independent square roots and use these to form invariants. Finally, we show that in normalized coordinates, the general reduced system of Sect. 4 reproduces the partial differential equation obtained in [18] for the case $M=S^{N-1}$ and in particular, the sine-Gordon equation for the case $M=S^{2}$.

\subsection{First Higher Conservation Law for the Grassmannian Model}

For the complex Grassmannian $s u(N) / S(U p) \times U(q))$, with $N=p+q$ and $p \leqq q$, the corresponding Lie algebra is the Lie algebra $s u(N)$ of traceless antihermitean complex $(N \times N)$-matrices, for which we shall use the following block matrix notation:

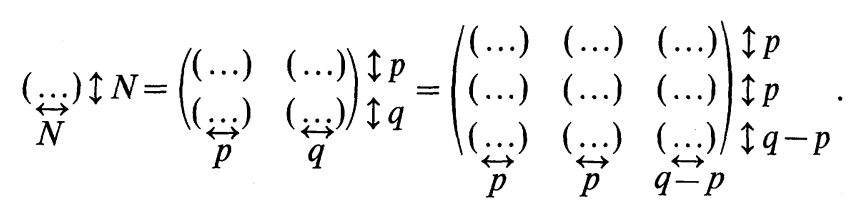


Then the decomposition (2.11) is given by

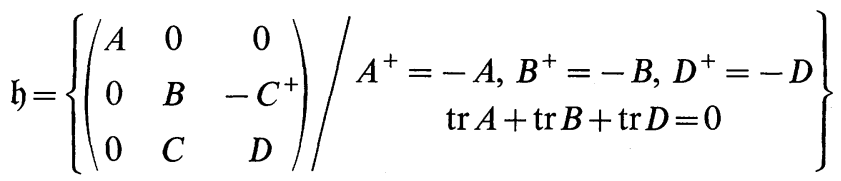

$$
\begin{aligned}
& \mathfrak{m}=\left\{\left(\begin{array}{ccc}
0 & -R^{+} & -S^{+} \\
R & 0 & 0 \\
S & 0 & 0
\end{array}\right)\right\}
\end{aligned}
$$

and

$$
\begin{gathered}
\mathfrak{I}=\left\{\left(\begin{array}{ccc}
i \Lambda & 0 & 0 \\
0 & i \Lambda & 0 \\
0 & 0 & D
\end{array}\right) / \begin{array}{c}
\Lambda \text { real diagonal, } D^{+}=-D \\
2 i \operatorname{tr} \Lambda+\operatorname{tr} D=0
\end{array}\right\} \\
\mathfrak{p}=\left\{\left(\begin{array}{ccc}
A & 0 & 0 \\
0 & B-A & -C^{+} \\
0 & C & 0
\end{array}\right) / A^{+}=-A, B^{+}=-B, \operatorname{diag}(B)=0\right\} \\
\mathfrak{a}=\left\{\left(\begin{array}{ccc}
0 & -\Lambda & 0 \\
\Lambda & 0 & 0 \\
0 & 0 & 0
\end{array}\right) / \Lambda \text { real diagonal }\right\} \\
\mathfrak{n}=\left\{\left(\begin{array}{ccc}
0 & -R^{+} & -S^{+} \\
R & 0 & 0 \\
S & 0 & 0
\end{array}\right) / \operatorname{diag}(R) \text { imaginary }\right\} .
\end{gathered}
$$

From [15, pp. 347-349], we also find

$$
\begin{aligned}
& \mathfrak{a}_{r}^{\circ}=\left\{\left(\begin{array}{ccc}
0 & -\Lambda & 0 \\
\Lambda & 0 & 0 \\
0 & 0 & 0
\end{array}\right) / \begin{array}{c}
\Lambda^{2} \text { real diagonal with nonzero and } \\
\text { mutually different entries }
\end{array}\right\} \\
& \mathfrak{a}^{+}=\left\{\left(\begin{array}{ccc}
0 & -\Lambda & 0 \\
\Lambda & 0 & 0 \\
0 & 0 & 0
\end{array}\right) / \Lambda \text { real diagonal with } \Lambda_{11}>\ldots>\Lambda_{p p}>0\right\},
\end{aligned}
$$

and therefore

$$
\mathfrak{m}_{r}^{\circ}=\left\{\left(\begin{array}{ccc}
0 & -R^{+} & -S^{+} \\
R & 0 & 0 \\
S & 0 & 0
\end{array}\right) / \begin{array}{c}
R^{+} R+S^{+} S \text { has nonzero and } \\
\text { mutually different eigenvalues }
\end{array}\right\} .
$$

Turning to the nonlinear $\sigma$-model on $S U(N) / S(U(p) \times U(q))$, we write the fields in the form $g=(X, Y)=\left(X, Y^{\prime}, Y^{\prime \prime}\right)$, where all matrices have $N$ rows and 
$g, X, Y, Y^{\prime}, Y^{\prime \prime}$ has $N, p, q, p, q-p$ columns, respectively (cf. [3, I] and (6.1)). Then as usual,

$$
D_{\mu} X=\partial_{\mu} X-X X^{+} \partial_{\mu} X, \quad D_{\mu} D_{v} X=\partial_{\mu} D_{v} X-D_{v} X X^{+} \partial_{\mu} X
$$

and

$$
\begin{gathered}
A_{\mu}=\left(\begin{array}{cc}
X^{+} \partial_{\mu} X & 0 \\
0 & Y^{+} \partial_{\mu} Y
\end{array}\right)=\left(\begin{array}{ccc}
X^{+} \partial_{\mu} X & 0 & 0 \\
0 & Y^{\prime+} \partial_{\mu} Y^{\prime} & Y^{+} \partial_{\mu} Y^{\prime \prime} \\
0 & Y^{\prime \prime}+\partial_{\mu} Y^{\prime} & Y^{\prime \prime} \partial_{\mu} Y^{\prime \prime}
\end{array}\right), \\
k_{\mu}=\left(\begin{array}{cc}
0 & -D_{\mu} X^{+} Y \\
Y^{+} D_{\mu} X & 0
\end{array}\right)=\left(\begin{array}{ccc}
0 & -D_{\mu} X^{+} Y^{\prime} & -D_{\mu} X^{+} Y^{\prime \prime} \\
Y^{\prime+} D_{\mu} X & 0 & 0 \\
Y^{\prime \prime} D_{\mu} X & 0 & 0
\end{array}\right) .
\end{gathered}
$$

Projecting out the various components corresponding to the above decomposition, we find

$$
\begin{aligned}
& \left(A_{\mu}\right)_{\mathrm{r}}=\left(\begin{array}{ccc}
\frac{1}{2} \operatorname{diag}\left(X^{+} \partial_{\mu} X+Y^{\prime+} \partial_{\mu} Y^{\prime}\right) & 0 & 0 \\
0 & \frac{1}{2} \operatorname{diag}\left(X^{+} \partial_{\mu} X+Y^{\prime+} \partial_{\mu} Y^{\prime}\right) & 0 \\
0 & 0 & Y^{\prime \prime} \partial_{\mu} Y^{\prime \prime}
\end{array}\right), \\
& \left(A_{\mu}\right)_{\mathfrak{p}}=\left(\begin{array}{ccc}
X^{+} \partial_{\mu} X-\frac{1}{2} \operatorname{diag}\left(X^{+} \partial_{\mu} X+Y^{\prime+} \partial_{\mu} Y^{\prime}\right) & 0 & 0 \\
0 & Y^{\prime+} \partial_{\mu} Y^{\prime}-\frac{1}{2} \operatorname{diag}\left(X^{+} \partial_{\mu} X+Y^{\prime+} \partial_{\mu} Y^{\prime}\right) & Y^{\prime+} \partial_{\mu} Y^{\prime \prime} \\
0 & Y^{\prime \prime} \partial_{\mu} Y^{\prime} & 0
\end{array}\right) \text {, } \\
& \left(k_{\mu}\right)_{\mathrm{a}}=\left(\begin{array}{ccc}
0 & -\operatorname{Re} \operatorname{diag}\left(D_{\mu} X^{+} Y^{\prime}\right) & 0 \\
\operatorname{Re} \operatorname{diag}\left(Y^{\prime+} D_{\mu} X\right) & 0 & 0 \\
0 & 0 & 0
\end{array}\right) \\
& \left(k_{\mu}\right)_{\mathfrak{r}}=\left(\begin{array}{ccc}
0 & -D_{\mu} X^{+} Y+\operatorname{Rediag}\left(D_{\mu} X^{+} Y\right) & -D_{\mu} X^{+} Y^{\prime \prime} \\
Y^{\prime+} D_{\mu} X-\operatorname{Rediag}\left(Y^{\prime+} D_{\mu} X\right) & 0 & 0 \\
Y^{\prime \prime} D_{\mu} X & 0 & 0
\end{array}\right) .
\end{aligned}
$$

For later use, we also introduce the notation

$$
\begin{gathered}
V=D_{\xi} X^{+} D_{\xi} X \\
W=\frac{1}{2}\left(D_{\xi} X^{+} D_{\xi} D_{\xi} X-D_{\xi} D_{\xi} X^{+} D_{\xi} X\right) .
\end{gathered}
$$

Now the PR gauge condition (4.1a) respectively (4.1b) means that

$$
k_{\xi}=\left(\begin{array}{ccc}
0 & -\kappa & 0 \\
\kappa & 0 & 0 \\
0 & 0 & 0
\end{array}\right) \text { i.e. } \quad Y^{\prime}=D_{\xi} X \kappa^{-1}
$$

with

$$
\kappa=V^{1 / 2}=\left(D_{\xi} X^{+} D_{\xi} X\right)^{1 / 2} \quad \text { real diagonal }
$$

and $\kappa$ such that the entries of $\kappa^{2}$ are nonzero and mutually different respectively that the entries of $\kappa$ satisfy $\kappa_{11}>\ldots>\kappa_{p p}>0$. This implies that in the PR gauge, 
$X^{+} \partial_{\eta} X$ and $X^{+} \partial_{\xi} X$ are both diagonal! In fact, for $X^{+} \partial_{\eta} X$ this follows directly from (6.8) and the equation $\left(A_{\eta}\right)_{\mathfrak{p}}=0$ [cf. (4.4)]. To prove the same for $X^{+} \partial_{\xi} X$, observe that

$$
\left[k_{\xi}, D_{\xi} k_{\xi}\right]=-\operatorname{ad}\left(k_{\xi}\right)^{2}\left(A_{\xi}\right)_{\mathfrak{p}}
$$

(because $\left[k_{\xi}, \partial_{\xi} k_{\xi}\right]=0$ and $\left.\left[\left(A_{\xi}\right)_{1}, k_{\xi}\right]=0\right)$. Writing this out in terms of $(3 \times 3)$ block matrices and expressing $Y^{\prime+} \partial_{\xi} Y^{\prime}$ in terms of $X$ and its derivatives [via (6.11) and (6.12)], we arrive at the equation

$$
\left[\kappa^{2}, X^{+} \partial_{\xi} X\right]=0
$$

which indeed implies that $X^{+} \partial_{\xi} X$ is diagonal.

Still working in the PR gauge, let us now write our first nontrivial local current [cf. (5.31)] in the form

$$
\begin{gathered}
\partial_{\xi} a^{(1)}=\frac{1}{2}\left[\operatorname{ad}\left(k_{\xi}\right)^{-1}\left(A_{\xi}\right)_{\mathfrak{p}},\left(A_{\xi}\right)_{\mathfrak{p}}\right]_{\mathfrak{a}}=\left(\begin{array}{ccc}
0 & -J_{\xi}^{(1)} & 0 \\
J_{\xi}^{(1)} & 0 & 0 \\
0 & 0 & 0
\end{array}\right), \\
\partial_{\eta} a^{(1)}=\left(k_{\eta}\right)_{\mathfrak{a}}=\left(\begin{array}{ccc}
0 & -J_{\eta}^{(1)} & 0 \\
J_{\eta}^{(1)} & 0 & 0 \\
0 & 0 & 0
\end{array}\right),
\end{gathered}
$$

where $J_{\xi}^{(1)}, J_{\eta}^{(1)}$ are fields taking values in the real diagonal $(p \times p)$-matrices. Then the entries $\left(J_{\xi}^{(1)}\right)_{j j}$ of $J_{\xi}^{(1)}$ and $\left(J_{\eta}^{(1)}\right)_{j j}$ of $J_{\eta}^{(1)}(1 \leqq j \leqq p)$ are explicitly given by

$$
\begin{aligned}
\left(J_{\xi}^{(1)}\right)_{j j}= & \frac{1}{2} \kappa_{j j}^{-3}\left(D_{\xi} D_{\xi} X^{+} D_{\xi} D_{\xi} X-\kappa^{4}-D_{\xi} D_{\xi} X^{+} D_{\xi} X \kappa^{-2} D_{\xi} X^{+} D_{\xi} D_{\xi} X\right)_{j j} \\
& -\frac{1}{2} \kappa_{j j}^{-1} \sum_{k=1}^{p} \tilde{\kappa}_{j k}^{-1} W_{j k} W_{k j}
\end{aligned}
$$

[cf. (6.10)] with

$$
\tilde{\kappa}_{j j}=\kappa_{j j}^{2}\left(2 \kappa_{j j}\right)^{2}, \quad \tilde{\kappa}_{j k}=\kappa_{k k}^{2}\left(\kappa_{j j}^{2}-\kappa_{k k}^{2}\right) \text { for } j \neq k,
$$

and

$$
\left(J_{\eta}^{(1)}\right)_{j j}=\frac{1}{2} \kappa_{j j}^{-1}\left(D_{\xi} X^{+} D_{\eta} X+D_{\eta} X^{+} D_{\xi} X\right)_{j j},
$$

respectively. In fact, (6.18) follows directly from (6.8). To prove (6.16), we make an Ansatz

$$
\operatorname{ad}\left(k_{\xi}\right)^{-1}\left(A_{\xi}\right)_{\mathfrak{p}}=\left(\begin{array}{ccc}
0 & -R^{+} \kappa^{-1} & -S^{+} \\
\kappa^{-1} R & 0 & 0 \\
S & 0 & 0
\end{array}\right),
$$

with $\operatorname{diag}(R)$ purely imaginary [cf. (6.3)], which implies

$$
\left(A_{\xi}\right)_{\mathfrak{p}}=\left(\begin{array}{ccc}
R^{+}-R & 0 & 0 \\
0 & \kappa^{-1} R \kappa-\kappa R^{+} \kappa^{-1} & -\kappa S^{+} \\
0 & S \kappa & 0
\end{array}\right) .
$$


Comparing this with (6.8) and using the fact that $X^{+} \partial_{\xi} X$ is diagonal, we derive that the diagonal entries $R_{j j}$ of $R(1 \leqq j \leqq p)$ and the offdiagonal entries $R_{j k}$ of $R$ $(1 \leqq j, k \leqq p, j \neq k)$ are given by

$$
R_{j j}=\left(2 \kappa_{j j}\right)^{-2} W_{j j}, \quad R_{j k}=\left(\kappa_{j j}^{2}-\kappa_{k k}^{2}\right)^{-1} W_{j k} \text { for } j \neq k
$$

[cf. (6.10)], and that

$$
S=Y^{\prime+} D_{\xi} D_{\xi} X \kappa^{-2} .
$$

Inserting this into the equation

$$
J_{\xi}^{(1)}=\frac{1}{2} \operatorname{Re} \operatorname{diag}\left(\kappa^{-1} R R^{+}-2 \kappa^{-1} R^{2}+\kappa R^{+} \kappa^{-2} R+\kappa S^{+} S\right)
$$

which follows from (6.15), (6.19), (6.20), we arrive at (6.16).

To compare our result with that of [12] (which contains some errors), we have to take the sum over $j$ and rearrange terms by using that

$$
\kappa_{j j}^{-1} \tilde{\kappa}_{j k}^{-1}+\kappa_{k k}^{-1} \tilde{\kappa}_{k j}^{-1}=\kappa_{j j}^{-2} \kappa_{k k}^{-2}\left(\kappa_{j j}+\kappa_{k k}\right)^{-1}
$$

for $1 \leqq j, k \leqq p$; this gives

$$
\begin{gathered}
\operatorname{tr}\left(J_{\xi}^{(1)}\right)=\frac{1}{2} \operatorname{tr} \kappa^{-3}\left(D_{\xi} D_{\xi} X^{+} D_{\xi} D_{\xi} X-\kappa^{4}-D_{\xi} D_{\xi} X^{+} D_{\xi} X \kappa^{-2} D_{\xi} X^{+} D_{\xi} D_{\xi} X\right) \\
-\frac{1}{4} \sum_{j, k=1}^{p} \kappa_{j j}^{-2} \kappa_{k k}^{-2}\left(\kappa_{j j}+\kappa_{k k}\right)^{-1} W_{j k} W_{k j}
\end{gathered}
$$

[cf. (6.10)], and

$$
\operatorname{tr}\left(J_{\eta}^{(1)}\right)=\frac{1}{2} \operatorname{tr} \kappa^{-1}\left(D_{\xi} X^{+} D_{\eta} X+D_{\eta} X^{+} D_{\xi} X\right) .
$$

From (6.16) (6.18) and (6.25), (6.26), we see again that the currents are invariant under residual gauge transformations, which act on the fields $X$ and $W$ according to $X \rightarrow X \exp (i \Lambda)$ and $W \rightarrow \exp (-i \Lambda) W \exp (i \Lambda)$, where $\Lambda$ is a field taking values in the real diagonal $(p \times p)$-matrices [cf. (6.3)], and that the regularity conditions ensure the denominators to be nonzero. It also follows that for $p=1$, our conservation law reduces to the usual conservation law for the complex projective space $\mathbb{C} P^{N-1}=S U(N) / S(U(1) \times U(N-1))[8]$, which, in contrast to the one for Grassmannians of higher $\operatorname{rank} p>1$, is automatically invariant under general gauge transformations.

To conclude, we want to show how for the rank2 Grassmannian $S U(N) / S(U(2) \times U(N-2))$, our conservation law can be rewritten in a form which is manifestly invariant under general gauge transformations. This is based on the observation that for any positive definite hermitean $(2 \times 2)$-matrix $V$ with inverse $V^{-1}$, we can give an explicit formula for the two linearly independent square roots $V_{+}^{1 / 2}, V_{-}^{1 / 2}$ of $V$ and $V_{+}^{-1 / 2}=\left(V_{+}^{1 / 2}\right)^{-1}, V_{-}^{-1 / 2}=\left(V_{-}^{1 / 2}\right)^{-1}$ of $V^{-18}$ :

$$
\begin{aligned}
V_{ \pm}^{1 / 2} & =\left(\operatorname{tr} V \pm 2(\operatorname{det} V)^{1 / 2}\right)^{-1 / 2}\left(V \pm(\operatorname{det} V)^{1 / 2}\right), \\
V_{ \pm}^{-1 / 2} & =\left(\operatorname{tr} V \pm 2(\operatorname{det} V)^{1 / 2}\right)^{-1 / 2}\left(1+(\operatorname{det} V)^{1 / 2} V^{-1}\right) .
\end{aligned}
$$

8 The index + , which is sometimes omitted [cf. (6.12)], is supposed to indicate that $V_{+}^{1 / 2}$ respectively $V_{+}^{-1 / 2}$ is the unique positive definite square root of $V$ respectively $V^{-1}$ 
Note that all these $(2 \times 2)$-matrices are again hermitean and commute with each other; moreover, denoting the eigenvalues of $V$ by $\lambda^{2}$ and $\mu^{2}$, with $\lambda \geqq \mu>0$, we get

$$
\lambda \pm \mu=\operatorname{tr} V_{ \pm}^{1 / 2} .
$$

For the proof, observe that we can always find a unitary $(2 \times 2)$-matrix $U \in U(2)$ such that

$$
U V U^{-1}=\left(\begin{array}{cc}
\lambda^{2} & 0 \\
0 & \mu^{2}
\end{array}\right)
$$

Then

$$
\operatorname{tr} V \pm 2(\operatorname{det} V)^{1 / 2}=(\lambda \pm \mu)^{2}
$$

and (6.27) reduces to the formulae

$$
\begin{aligned}
U V_{ \pm}^{1 / 2} U^{-1} & =\left(\begin{array}{cc}
\lambda & 0 \\
0 & \pm \mu
\end{array}\right), \\
U V_{ \pm}^{-1 / 2} U^{-1} & =\left(\begin{array}{cc}
\lambda^{-1} & 0 \\
0 & \pm \mu^{-1}
\end{array}\right),
\end{aligned}
$$

from which all our statements are obvious; note that an explicit knowledge of $U$ is not required.

Now with $V$ given by (6.9), we can rewrite our first nontrivial local current in the form

$$
\begin{aligned}
& \left(J_{\xi}^{(1)}\right)_{11} \pm\left(J_{\xi}^{(1)}\right)_{22} \\
& \quad=\frac{1}{2} \operatorname{tr} V_{ \pm}^{-3 / 2}\left(D_{\xi} D_{\xi} X^{+} D_{\xi} D_{\xi} X-V^{2}-D_{\xi} D_{\xi} X^{+} D_{\xi} X V^{-1} D_{\xi} X^{+} D_{\xi} D_{\xi} X\right) \\
& \quad-\frac{1}{8}\left(\operatorname{tr} V_{ \pm}^{1 / 2}\right)^{-1}\left\{\operatorname{tr}\left(V^{-1} W\right)^{2}+\left(\operatorname{det} V_{ \pm}^{1 / 2}\right) \operatorname{tr}\left(V_{ \pm}^{-3 / 2} W\right)^{2}\right\}
\end{aligned}
$$

[cf. (6.10)], and

$$
\left(J_{\eta}^{(1)}\right)_{11} \pm\left(J_{\eta}^{(1)}\right)_{22}=\frac{1}{2} \operatorname{tr} V_{ \pm}^{-1 / 2}\left(D_{\xi} X^{+} D_{\eta} X+D_{\eta} X^{+} D_{\xi} X\right) .
$$

These expressions are obviously invariant under general gauge transformations, which act on the fields $X, V, V_{ \pm}^{k / 2}(k \in \mathbb{Z})$ and $W$ according to $X \rightarrow X \tilde{h}$, $V \rightarrow \tilde{h}^{-1} V \tilde{h}, V_{ \pm}^{k / 2} \rightarrow \tilde{h}^{-1} V_{ \pm}^{k / 2} \tilde{h}\left[k \in \mathbb{Z}\right.$; cf. (6.27)] and $W \rightarrow \tilde{h}^{-1} W \tilde{h}$, where $\tilde{h}$ is a field taking values in $U(2)$, and a short calculation shows that in the PR gauge, (6.29) is equivalent to (6.16) and (6.30) is equivalent to (6.18).

Of course, the entire analysis of this section also applies, mutatis mutandis, to the real Grassmannian $S O(N) / S O(p) \times S O(q)$ with $N=p+q$ and $p \leqq q$. In fact, all we have to do is to replace + (hermitean adjoint) by $T$ (transpose) and discard all imaginary parts; we leave the details of these modifications to the reader.

\subsection{Reduced System for the Sphere Model}

To show that for the nonlinear $\sigma$-model on the sphere $S^{N-1}=S O(N) / S O(N-1)$, our general definition (4.11)-(4.13) of the reduced system reproduces the result of 
[18], and in particular, for $N=3$, the sine-Gordon equation, we write

$$
\begin{array}{rlrl}
k_{\xi} & =\left(\begin{array}{ccc}
0 & -\kappa & 0 \\
\kappa & 0 & 0 \\
0 & 0 & 0
\end{array}\right), & \phi^{R}=\left(\begin{array}{ccc}
0 & 0 & -\boldsymbol{\phi}^{\top} \\
0 & 0 & 0 \\
\boldsymbol{\phi} & 0 & 0
\end{array}\right), \\
\left(k_{\eta}\right)_{\mathfrak{a}}=\left(\begin{array}{ccc}
0 & -\beta & 0 \\
\beta & 0 & 0 \\
0 & 0 & 0
\end{array}\right), & \left(k_{\eta}^{R}\right)_{\mathfrak{n}}=\left(\begin{array}{ccc}
0 & 0 & -\boldsymbol{\varphi}^{\top} \\
0 & 0 & 0 \\
\boldsymbol{\varphi} & 0 & 0
\end{array}\right),
\end{array}
$$

and

$$
\lambda=\sqrt{\beta^{2}+\varphi^{2}}, \quad \beta=\lambda \cos \alpha, \quad \boldsymbol{\varphi}=\lambda \sin \alpha \mathbf{f},
$$

where $\kappa, \lambda, \alpha, \beta$ and $\phi, \varphi, f$ are fields taking values in $\mathbb{R}$ and in $\mathbb{R}^{N-2}$, respectively, with $\kappa>0, \lambda>0$, and $\mathbf{f}^{2}=1$; observe that in terms of the original field $q[1,18]$, the variables $\kappa, \lambda$, and $\alpha$ are given by

$$
\kappa=\left\|\partial_{\xi} q\right\|, \quad \lambda=\left\|\partial_{\eta} q\right\|, \quad \cos \alpha=\frac{\partial_{\xi} q \cdot \partial_{\eta} q}{\left\|\partial_{\xi} q\right\|\left\|\partial_{\eta} q\right\|} .
$$

Then $\varphi=\partial_{\eta} \phi$ [cf. (4.4)], and the differential equations (4.11)-(4.13) take the form

$$
\begin{gathered}
\partial_{\eta} \kappa=0, \\
\partial_{\eta}\left(\frac{1}{2} \kappa \phi^{2}\right)=\partial_{\xi} \beta, \\
\square \phi+\kappa \beta \phi=0,
\end{gathered}
$$

implying

$$
\partial_{\xi} \lambda=0
$$

In terms of normalized coordinates, we have $\kappa=1, \lambda=1$ [cf. (6.33)], and $\varphi$ solves the equation

$$
\partial_{\eta}\left(\left(1-\varphi^{2}\right)^{-1 / 2} \partial_{\xi} \varphi\right)+\varphi=0
$$

obtained in [18]. For $N=3$, this reduces to the statement that $\alpha$ solves the sineGordon equation

$$
\square \alpha+\sin \alpha=0 \text {. }
$$

\section{References}

1. Pohlmeyer, K.: Commun. Math. Phys. 46, 207 (1976)

2. Eichenherr, H.: Nucl. Phys. B 146, 215 (1978); B 155, 544 (1979)

Golo, V.I., Perelomov, A.M.: Lett. Math. Phys. 2, 477 (1978)

3. Eichenherr, H., Forger, M.: Nucl. Phys. B 155, 381 (1979); Nucl. Phys. B 164, 528 (1980)

4. Forger, M.: Instantons in nonlinear $\sigma$-models, gauge theories and general relativity. In: Differential geometric methods in mathematical physics. Proceedings, Clausthal, Germany 1978. In: Lecture Notes in Physics, Vol. 139, Berlin, Heidelberg, New York: Springer 1981 
5. Maison, D.: Some facts about classical nonlinear $\sigma$-models. Max-Planck-Institute preprint MPIPAE/PTh 52/79, to appear in the Proceedings of the $16^{\text {th }}$ Winter School of Theoretical Physics, Karpacz 1980

6. Forger, M.: PhD Thesis, Freie Universität Berlin (unpublished)

7. Lüscher, M., Pohlmeyer, K.: Nucl. Phys. B 137, 46 (1978)

8. Eichenherr, H.: Phys. Lett. 90 B, $121(1980)$

9. Cherednik, I.V.: Theor. Math. Phys. 38, 120 (1979); Theor. Math. Phys. 41, 997 (1979)

10. Ogielski, A.T., Prasad, M.-K., Sinha, A., Chau Wang, L.L.: Phys. Lett. 91 B, 387 (1980)

11. Scheler, K.: Phys. Lett. 93 B, 331 (1980); Z. Phys. C - Particles and Fields 6, 365 (1980)

12. Chou, K.C., Song, X.C.: Bäcklund transformation, local and nonlocal conservation laws for nonlinear $\sigma$-models on symmetric coset spaces and Local conservation laws for various nonlinear $\sigma$-models. Academica Sinica preprints ASITP 80-008 and 80-010 (1980)

13. Caselle, M., Megna, R., Sciuto, S.: Generalizations of the sine-Gordon equation with $S U(p+q) / S(U(p) \times U(q))$ structure. University of Torino preprint IFTT 402 January 1981

14. Eichenherr, H., Honerkamp, J.: J. Math. Phys. 22, 374 (1981)

15. Helgason, S. : Differential geometry, Lie groups, and symmetric spaces. New York : Academic Press 1978

16. Kobayashi, S., Nomizu, K. : Foundations of differential geometry, Vol. 2. New York: Interscience 1969

17. Mack, G. : Fortschr. d. Phys. 29, 135 (1981)

18. Pohlmeyer, K., Rehren, K.H.: J. Math. Phys. 20, 2628 (1979)

19. D’Auria, R., Regge, T., Sciuto, S.: Phys. Lett. 89 B, 363 (1980); Nucl. Phys. B 171, 167 (1980)

20. Golo, V.I., Putko, B.A.: Lett. Math. Phys. 4, 195 (1980)

Communicated by R. Stora

Received May 8, 1981 
